\title{
Robust feedback control of Rayleigh-Bénard convection
}

\author{
By A. C. OR ${ }^{1}$, L. CORTELEZZI ${ }^{2}$ AND J. L. SPEYER ${ }^{1}$ \\ ${ }^{1}$ Department of Mechanical \& Aerospace Engineering, University of California, \\ Los Angeles, CA 90095-1597, USA \\ ${ }^{2}$ Department of Mechanical Engineering, McGill University, Montreal, Canada H3A 2K6
}

(Received 28 July 2000 and in revised form 7 December 2000)

We investigate the application of linear-quadratic-Gaussian (LQG) feedback control, or, in modern terms, $\mathscr{H}_{2}$ control, to the stabilization of the no-motion state against the onset of Rayleigh-Bénard convection in an infinite layer of Boussinesq fluid. We use two sensing and actuating methods: the planar sensor model (Tang \& Bau 1993, 1994), and the shadowgraph model (Howle 1997a). By extending the planar sensor model to the multi-sensor case, it is shown that a LQG controller is capable of stabilizing the no-motion state up to 14.5 times the critical Rayleigh number. We characterize the robustness of the controller with respect to parameter uncertainties, unmodelled dynamics. Results indicate that the LQG controller provides robust performances even at high Rayleigh numbers.

\section{Introduction}

When a layer of fluid at rest is heated from below, fluid motion will develop into well-organized convection patterns if the temperature difference across the layer is sufficiently large (Cross \& Hohenberg 1993). For certain industrial applications, developing a temperature gradient across the fluid layer is unavoidable but at the same time preventing convective fluid motions is desirable. Some examples involving undesirable effects of convection are materials processing, solidification, semiconductor melts, welding, evaporative coating and crystal growth. Our aim is to use robust modern control methodologies to inhibit the onset of convection while permitting a large thermal gradient across the layer of fluid.

The idea of stabilizing the fluid layer against the onset of cellular motions has been advanced by Tang \& Bau (1993, 1994, 1998a,b) and Howle (1997a-c, 2000). Tang \& Bau assumed that the temperature field can be measured continuously on a horizontal plane in $x, y$ and $t$ (see figure 1). The measurements are then used to control the temperature at the lower wall, in order to cancel the thermal disturbances in the fluid that drive the overturning motions. Howle (1997a) investigated a similar control problem, except in his case the measurements consist of shadowgraph images of the fluid. The shadowgraph images capture the horizontal distribution of the verticalmean temperature. Moreover, in Howle's model, heat flux rather than temperature is prescribed at the lower wall. Both types of sensor and actuator models will be considered in this study using a more sophisticated form of control synthesis.

Based on proportional feedback control, the results of Tang \& Bau and Howle show that both sensor models exhibit a maximal achievable stable value of Rayleigh number $R a$, beyond which this simple controller is ineffective for stabilization. For the 


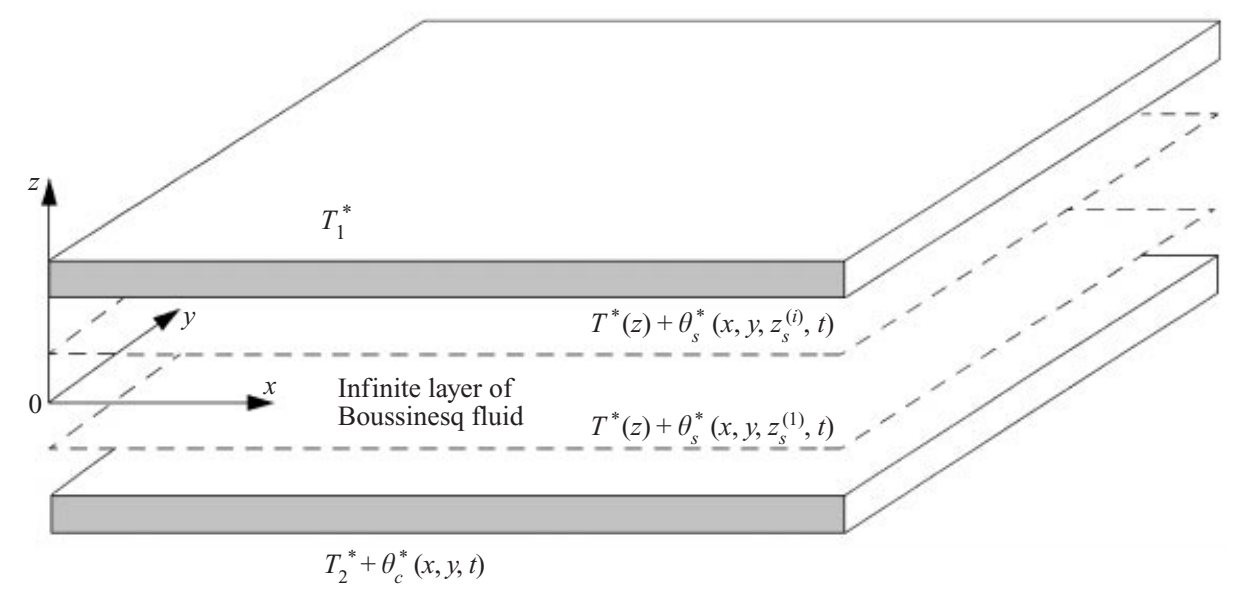

Figure 1. Planar sensor model.

planar-sensor model Tang \& Bau $(1993,1994)$ showed that the controller can inhibit convection up to a maximum of about 3.8 times $R a_{c 0}$ at $P r=7$, where $R a_{c 0}=1707.76$ is the uncontrolled critical value. This value is $\operatorname{Pr}$-dependent. Furthermore, they (Tang $\&$ Bau 1994) considered a velocity actuator which inhibits convection up to 10 times the critical Rayleigh number. For the shadowgraph sensor model this maximum is about 3.13 times $R a_{c 0}=1295.78$. In $\S 4$ the differences in performance between the two sensor models using only the thermal actuators, and the limitations of the proportional feedback control will be discussed.

The degree of stabilization can be improved significantly by using synthesis methods for multiple-input/multiple-output systems which produce dynamic compensators. However, in this study a distributed single-input/multiple-output strategy will be considered, since normal modes of different wavenumbers decouple and each normal mode can be controlled individually.

One such synthesis method is known as the linear-quadratic-Gaussian (LQG) control (Bryson \& Ho 1969), or, in modern terms, $\mathscr{H}_{2}$ control. Other methods such as $\mathscr{H}_{\infty}$ synthesis (Rhee \& Speyer 1991) could also be applied, but we suspect with similar results. The LQG approach allows us to consider a nominal design Rayleigh number $\left(R a^{*}\right)$ significantly higher than that considered in the previous studies. For values of $R a$ sufficiently near $R a^{*}$, stabilization with the LQG controller appears always to be achievable. Moreover, for $R a^{*}$ below a certain threshold, the complete range of $R a$ up to a critical value can be stabilized. In this study, we show that the system can be stabilized up to $R a=14.8 R a_{c 0}$. In this range the first even and odd modes of convection can become unstable. However, these two modes are damped by the LQG controller. The higher modes are naturally damped within this range of Ra. Our aim is to ensure stability over the entire range of $R a$ up to a maximum critical Rayleigh number, without the formation of isolated unstable regions within this range. This requires the determination of the value of $R a^{*}$ to produce this maximum critical Rayleigh number.

Unlike the proportional control method, the LQG synthesis method requires some elaboration. This synthesis method consists of two steps: (i) a reconstruction of the internal states of the plant based on the measured information, and (ii) a regulation of the plant states in order to drive the estimated perturbations to a zero level. To 
accomplish these two steps, the LQG controller is formed by a Kalman filter and an optimal regulator in cascade.

The LQG synthesis method has recently been used in the study of drag reduction in channel flow by Joshi, Speyer \& Kim (1999) and by Cortelezzi \& Speyer (1998) based on Joshi, Speyer \& Kim (1997) who first introduced the system theory approach. Cortelezzi \& Speyer (1998) presented a framework suited to practical implementations and demonstrated the performance of the design in numerical simulations. Following this framework, our investigations focus on a robust stability analysis of the closedloop system as applied to Rayleigh-Bénard convection. Design parameters of the filter and regulator are carefully chosen to enhance the robustness of the stabilization. Several integrated design aspects are discussed.

This study is intended to provide a design of controllers for future experiments. The design goal is to maximize the stability range of Rayleigh number. This implies designing controllers at the highest possible design value $R a^{*}$, without causing an unstable, isolated region to form below $R a^{*}$. Since the controllers are implemented at each wavenumber and only $R a$ is being varied, one form of robustness being demonstrated is the robustness of the system with respect to variations in $R a$ away from $R a^{*}$. Another form of robustness is concerned with uncertainties in the system apart from the mismatch in $R a$, for example uncertainties due to nonlinearities or unmodelled dynamics. The objective of this paper is to produce a robust design based on classical relative stability measures of gain and phase margins that accommodates to a degree unmodelled dynamics and nonlinearities. Nonlinear simulation is required to validate the design. We will show that the gain and phase margins depends crucially on the number of sensors used. More measurements implies better knowledge about the internal states of the system. In this study we are interested in determining the minimum number of sensor planes, as well as their locations, for achieving a reasonable degree of robustness. From a theoretical point of view, the planar sensor model appears to be more effective and accurate than the shadowgraph sensor model, mainly because a multiple planar sensor configuration can be readily incorporated. As the results will show, increasing the number of sensors, i.e. the measured information about the internal states, is crucial for achieving the desirable robustness.

We now proceed to $\S 2$ to derive the standard state-space equations for both sensor models. In $\S 3$, we review the theory of the LQG optimal control synthesis. In $\S 4$, the results are discussed and in $\S 5$, we conclude the paper.

\section{State-space formulation}

In this Section, we derive the state-space equations. Following the framework presented by Cortelezzi \& Speyer (1998), we start from the governing equations and subsequently transform them into a set of ordinary differential equations expressed in state-space form. This procedure includes a transformation of variables, a spectral decomposition of the resulting equations and expressing these equations in the standard state-space form.

\subsection{Governing equations}

We consider an infinite layer of Boussinesq fluid heated from below, which is parallel to the $(x, y)$-plane and bounded by non-permeable walls at $z= \pm d / 2$ (see figure 1). Our aim is to compare the performance of the LQG controller with the performance of the proportional feedback control method based on two known models. Therefore, in our formulation the boundary conditions used in these two models will be preserved. 
For both models, the upper wall is assumed to be infinitely conductive at constant temperature $T_{1}^{*}$. For the planar sensor model (Tang \& Bau 1993, 1994, 1998a,b) temperature is measured on an interior plane (see figure $1, T^{*}(z)$ denotes the basic state). These measurements are used to modify the constant lower wall temperature $T_{2}^{*}$ with the actuator temperature $\theta_{c}^{*}(x, y, t)$. For the shadowgraph sensor model (Howle $1997 a, b)$ measurements of the vertical-mean temperature distribution are obtained in the form of a shadowgraph. These measurements are used to modify the constant heat flux $Q^{*}$ at the lower wall with the actuator heat flux $q_{c}^{*}(x, y, t)$. Different actuators are used to allow direct comparisons between the performances of the LQG controllers and the proportional controllers used in the original models.

We scale length, time, velocity, pressure relative to the hydrostatic pressure, and temperature, respectively, by $d, d^{2} / \kappa, \kappa / d, \rho v \kappa / d^{2}$ and $\left(T_{2}^{*}-T_{1}^{*}\right)$ or $Q^{*} d / K$ depending on the model chosen, where $\rho, \kappa, v$ and $K$ are, respectively, density, thermal diffusivity, kinematic viscosity and thermal conductivity of the fluid. The linear stability equations for the vertical perturbation velocity $w(x, y, z, t)$ and perturbation temperature $\theta(x, y, z, t)$ are

$$
\begin{gathered}
\left(\operatorname{Pr}^{-1} \partial_{t}-\nabla^{2}\right) \nabla^{2} w=R a \nabla_{\perp}^{2} \theta, \\
\left(\partial_{t}-\nabla^{2}\right) \theta=w,
\end{gathered}
$$

where $\nabla_{\perp}^{2}=\nabla^{2}-\partial_{z z}$ (e.g. Chandrashekhar 1961). The Prandtl number is $\operatorname{Pr}=v / \kappa$. The Rayleigh number for the planar and shadowgraph cases are defined, respectively, as $R a=\alpha g\left(T_{2}^{*}-T_{1}^{*}\right) d^{3} / v \kappa$ and $R a=\alpha g Q^{*} d^{4} / K v \kappa$, where $\alpha$ is the coefficient of volume expansion and $g$ is the gravitational acceleration.

The boundary conditions on velocity are

$$
w(x, y, \pm 1 / 2, t)=\partial_{z} w(x, y, \pm 1 / 2, t)=0 .
$$

For the thermal boundary conditions, the upper wall is considered isothermal so that the perturbation temperature must satisfy

$$
\theta(x, y, 1 / 2, t)=0 .
$$

Based on the planar and shadowgraph sensor models (Tang \& Bau 1993, 1994; Howle 1997a), we apply a continuous time-dependent control temperature along the lower wall for the planar sensor case,

$$
\theta(x, y,-1 / 2, t)=\theta_{c}(x, y, t),
$$

while for the shadowgraph model we apply a continuous time-dependent heat flux $q_{c}$ instead,

$$
\partial_{z} \theta(x, y,-1 / 2, t)=q_{c}(x, y, t) .
$$

The planar sensor model measures the temperature distribution at a number $I$ of $(x, y)$-planes located at $z=z_{s}^{(i)} \in[-1 / 2,1 / 2]$, where $i=1,2, \ldots, I$. The measurement equations are

$$
\theta_{s}^{(i)}(x, y, t)=\theta\left(x, y, z_{s}^{(i)}, t\right), \quad i=1,2, \ldots, I,
$$

where $z_{s}^{(i)}$ is the $z$-coordinate of the $i$ th sensor plane. The shadowgraph model measures the average density over the whole layer, $\rho_{s}$, which is expressed in terms of temperature by

$$
\rho_{s}(x, y, t)=\int_{-1 / 2}^{1 / 2} \nabla_{\perp}^{2} \theta(x, y, z, t) \mathrm{d} z
$$


where $\rho_{s}$ is the measurement function in density. We refer readers to Howle (1997a) for the derivations of this integral expression.

\subsection{Transformation of dependent variables and cost criterion}

We transform the perturbation temperature so that equations (2.1)-(2.8) have homogeneous thermal boundary conditions. The perturbation velocity remains unchanged. With the perturbation temperature as $\theta \equiv \phi+\xi$, we obtain for both sensor models

$$
\begin{gathered}
\left(P r^{-1} \partial_{t}-\nabla^{2}\right) \nabla^{2} w-R a \nabla_{\perp}^{2} \phi=R a \nabla_{\perp}^{2} \xi, \\
\left(\partial_{t}-\nabla^{2}\right) \phi-w=-\left(\partial_{t}-\nabla^{2}\right) \xi,
\end{gathered}
$$

subject to the boundary conditions

$$
w(x, y, \pm 1 / 2, t)=\partial_{z} w(x, y, \pm 1 / 2, t)=\phi(x, y, 1 / 2, t)=0 .
$$

Furthermore, for the planar sensor model $\phi$ must satisfy the boundary condition

$$
\phi(x, y,-1 / 2, t)=0,
$$

while for the shadowgraph sensor model $\phi$ must satisfy the boundary condition

$$
\partial_{z} \phi(x, y,-1 / 2, t)=0 .
$$

The forcing function $\xi$ satisfies a non-homogeneous boundary condition at the lower wall and a homogeneous boundary condition at the upper wall. For the planar case these conditions are

$$
\xi(x, y,-1 / 2, t)=\theta_{c}(x, y, t), \quad \xi(x, y, 1 / 2, t)=0,
$$

and for the shadowgraph case we have instead

$$
\partial_{z} \xi(x, y,-1 / 2, t)=q_{c}(x, y, t), \quad \xi(x, y, 1 / 2, t)=0 .
$$

The two set of measurement equations in terms of the new variables for the planar and shadowgraph cases become

$$
\theta_{s}^{(i)}(x, y, t)=\phi\left(x, y, z_{s}^{(i)}, t\right)+\xi\left(x, y, z_{s}^{(i)}, t\right), \quad i=1,2, \ldots, I,
$$

and

$$
\rho_{s}(x, y, t)=\int_{-1 / 2}^{1 / 2} \nabla_{\perp}^{2}(\phi(x, y, z, t)+\xi(x, y, z, t)) \mathrm{d} z .
$$

Note that the sensors could be located at discrete points in the $(x, y)$-plane, but to be consistent with Tang \& Bau (1993, 1994) we have considered continuously distributed sensors. Furthermore, Tang \& Bau's (1998a) experiment using discrete sensors and actuators shows consistency with their theoretical work using continuously distributed sensors and actuators.

Finally, we introduce the cost criterion. Our goal is to design a controller able to drive the measured perturbation temperature to zero, without using unnecessarily large control action, hopefully resulting in little saturation of the actuators. Thus, the performance index includes weighting on the control. We consider a layer of fluid with large aspect ratios $L_{x}$ and $L_{y}$ with periodic boundary conditions (see figure 1), assuming that the influence of the lateral boundary conditions in a finite layer of fluid is negligible. The LQG controller is determined by finding the control action which minimizes the cost criterion. For the planar sensor model we define the cost criterion 
as

$$
\mathscr{J}=\int_{t}^{T}\left\{\int_{0}^{L_{x}} \int_{0}^{L_{y}}\left[\sum_{i=1}^{I}\left(\theta_{s}^{(i)}\right)^{2}+\gamma \theta_{c}^{2}\right] \mathrm{d} x \mathrm{~d} y\right\} \mathrm{d} \tau,
$$

and for the shadowgraph sensor model it is

$$
\mathscr{J}=\int_{t}^{T}\left\{\int_{0}^{L_{x}} \int_{0}^{L_{y}} \int_{t}^{T}\left(\rho_{s}^{2}+\gamma q_{c}^{2}\right) \mathrm{d} x \mathrm{~d} y\right\} \mathrm{d} \tau
$$

\subsection{Modal decomposition}

A periodic boundary condition permits us to perform a Fourier decomposition in the horizontal coordinates. The vertical dependence of the flow field and thermal field is constrained by the upper and lower wall boundary conditions. The vertical dependence will be decomposed separately in $\S 2.4$. We describe an infinitesimal threedimensional disturbance to the no-motion state. Consequently, we have a double sum of the Fourier normal modes for the disturbances:

$$
\left.\begin{array}{l}
w(x, y, z, t)=\sum_{m=1}^{M} \sum_{n=1}^{N}\left\{\frac{1}{2}\left[W_{r, m n}(z, t)+\mathrm{i} W_{i, m n}(z, t)\right] \mathrm{e}^{\mathrm{i}\left(m k_{x} x+n k_{y} y\right)}+\text { c.c. }\right\}, \\
\phi(x, y, z, t)=\sum_{m=1}^{M} \sum_{n=1}^{N}\left\{\frac{1}{2}\left[\Phi_{r, m n}(z, t)+\mathrm{i} \Phi_{i, m n}(z, t)\right] \mathrm{e}^{\mathrm{i}\left(m k_{x} x+n k_{y} y\right)}+\text { c.c. }\right\}, \\
\xi(x, y, z, t)=\sum_{m=1}^{M} \sum_{n=1}^{N}\left\{\frac{1}{2}\left[\Xi_{r, m n}(z, t)+\mathrm{i} \Xi_{i, m n}(z, t)\right] \mathrm{e}^{\mathrm{i}\left(m k_{x} x+n k_{y} y\right)}+\text { c.c. }\right\},
\end{array}\right\}
$$

where c.c. denotes the complex conjugate. The measurement and control functions are represented by

$$
\begin{aligned}
& \theta_{s}^{(j)}(x, y, t)=\sum_{m=1}^{M} \sum_{n=1}^{N}\left\{\frac{1}{2}\left[\Theta_{s r, m n}^{(j)}(t)+\mathrm{i} \Theta_{s i, m n}^{(j)}(t)\right] \mathrm{e}^{\mathrm{i}\left(m k_{x} x+n k_{y} y\right)}+\text { c.c. }\right\}, \quad j=1, \ldots, I, \\
& \rho_{s}(x, y, t)=\sum_{m=1}^{M} \sum_{n=1}^{N}\left\{\frac{1}{2}\left[R_{s r, m n}(t)+\mathrm{i} R_{s i, m n}(t)\right] \mathrm{e}^{\mathrm{i}\left(m k_{x} x+n k_{y} y\right)}+\text { c.c. }\right\} \\
& \theta_{c}(x, y, t)=\sum_{m=1}^{M} \sum_{n=1}^{N}\left\{\frac{1}{2}\left[\Theta_{c r, m n}(t)+\mathrm{i} \Theta_{c i, m n}(t)\right] \mathrm{e}^{\mathrm{i}\left(m k_{x} x+n k_{y} y\right)}+\text { c.c. }\right\} \\
& q_{c}(x, y, t)=\sum_{m=1}^{M} \sum_{n=1}^{N}\left\{\frac{1}{2}\left[Q_{c r, m n}(t)+\mathrm{i} Q_{c i, m n}(t)\right] \mathrm{e}^{\mathrm{i}\left(m k_{x} x+n k_{y} y\right)}+\text { c.c. }\right\}
\end{aligned}
$$

where the subscripts $r$ and $i$ indicate real and imaginary parts, respectively. The two fundamental wavenumbers are $k_{x}=2 \pi / L_{x}$ and $k_{y}=2 \pi / L_{y}$. From the classical theory without control, a normal mode disturbance is unstable in the region above a neutral curve $R a_{0}(k)$ (Chandrashekhar 1961), where $R a_{0}$ is the Rayleigh number at neutral stability in the open-loop system and $k=\sqrt{k_{x}^{2}+k_{y}^{2}}$ is the magnitude of the wavevector. We truncate the infinite series above to $M \times N$ horizontal modes, which span the unstable range. Since the basic equations depend only on the horizontal Laplacian $\nabla_{\perp}^{2}$, the wavenumbers appear only in even powers and can be described 
in terms of an internal parameter $k(m, n)$, where $k(m, n)=\sqrt{m^{2} k_{x}^{2}+n^{2} k_{y}^{2}}$. We further substitute (2.20) and (2.21) into equations (2.9)-(2.17) and separate the real and imaginary parts. It should be remarked that the linear structure includes all patterns at the onset. Certain realizable patterns, such as rolls and hexagons, will be selected when the nonlinear and symmetry-breaking effects are included in the model. The paper is focused on the stabilization of the no-motion state. Suppression of selected convection patterns and return to the no-motion state will be addressed by applying our LQG controller to a direct numerical simulation of convection.

Since the governing equations contain only even derivatives with respect to $x$ or $y$, the real and imaginary parts of the dependent variables decouple and satisfy the same set of equations. Furthermore, since the problem is linear, we can consider each normal mode separately. For simplicity of notation, we drop the indices of the Fourier coefficients, and define $W \equiv W_{r, m n}=W_{i, m n}, \Phi \equiv \Phi_{r, m n}=\Phi_{i, m n}, \Xi \equiv \Xi_{r, m n}=\Xi_{i, m n}, \Theta_{c} \equiv$ $\Theta_{c r, m n}=\Theta_{c i, m n}, Q_{c} \equiv Q_{c r, m n}=Q_{c i, m n}, \Theta_{s}^{(i)} \equiv \Theta_{s r, m n}^{(i)}=\Theta_{s i, m n}^{(i)}$ and $R_{s} \equiv R_{s r, m n}=R_{s i, m n}$. The governing equations are reduced as follows:

$$
\begin{gathered}
{\left[\operatorname{Pr}^{-1}\left(\partial_{z}^{2}-k^{2}\right) \partial_{t}-\left(\partial_{z}^{2}-k^{2}\right)^{2}\right] W+R a k^{2} \Phi=-R a k^{2} \Xi,} \\
{\left[\partial_{t}-\left(\partial_{z}^{2}-k^{2}\right)\right] \Phi-W=-\left[\partial_{t}-\left(\partial_{z}^{2}-k^{2}\right)\right] \Xi .}
\end{gathered}
$$

The boundary conditions are homogeneous. For the planar case we have

$$
W( \pm 1 / 2, t)=\partial_{z} W( \pm 1 / 2, t)=\Phi( \pm 1 / 2, t)=0,
$$

and for the shadowgraph sensor model we have instead

$$
W( \pm 1 / 2, t)=\partial_{z} W( \pm 1 / 2, t)=\Phi(1 / 2, t)=0, \quad \partial_{z} \Phi(-1 / 2, t)=0 .
$$

The forcing function $\Xi$ satisfies the non-homogeneous boundary condition at the lower wall and the homogeneous boundary condition at the upper wall. For the planar case the forcing function is given by

$$
\Xi(-1 / 2, t)=\Theta_{c}(t), \quad \Xi(1 / 2, t)=0,
$$

and the corresponding measurement functions are

$$
\Theta_{s}^{(i)}(t)=\Phi\left(z_{s}^{(i)}, t\right)+\Xi\left(z_{s}^{(i)}, t\right), \quad i=1,2, \ldots, I .
$$

For the shadowgraph sensor model, the forcing function $\Xi$ is

$$
\partial_{z} \Xi(-1 / 2, t)=Q_{c}(t), \quad \Xi(1 / 2, t)=0,
$$

and the corresponding measurement function is

$$
R_{s}(t)=-k^{2} \int_{-1 / 2}^{1 / 2}(\Phi(z, t)+\Xi(z, t)) \mathrm{d} z
$$

In our approach, each distinct horizontal normal mode is controlled by a separate controller. Therefore, for the implementation $M \times N$ controllers are required. As a simple illustration for the analysis, consider an aspect ratio $L_{x} / d$ and $L_{y} / d$ equal to $20 \pi$. In this case, the only wavenumbers present are the fundamental wavenumber $k_{f}=2 \pi / L=0.05$, and its harmonics: for $R a$ up to $15 R a_{c 0}$, the wavenumbers are from $k=k_{f}$ to $k=12$ (equal to $240 k_{f}$ ). These wavenumbers represent the ensemble of normal modes used to represent the convection field.

In a physical implementation of the planar sensor model, both the measurements and control action occur in physical space but the controllers operate in the Fourier 
(a)

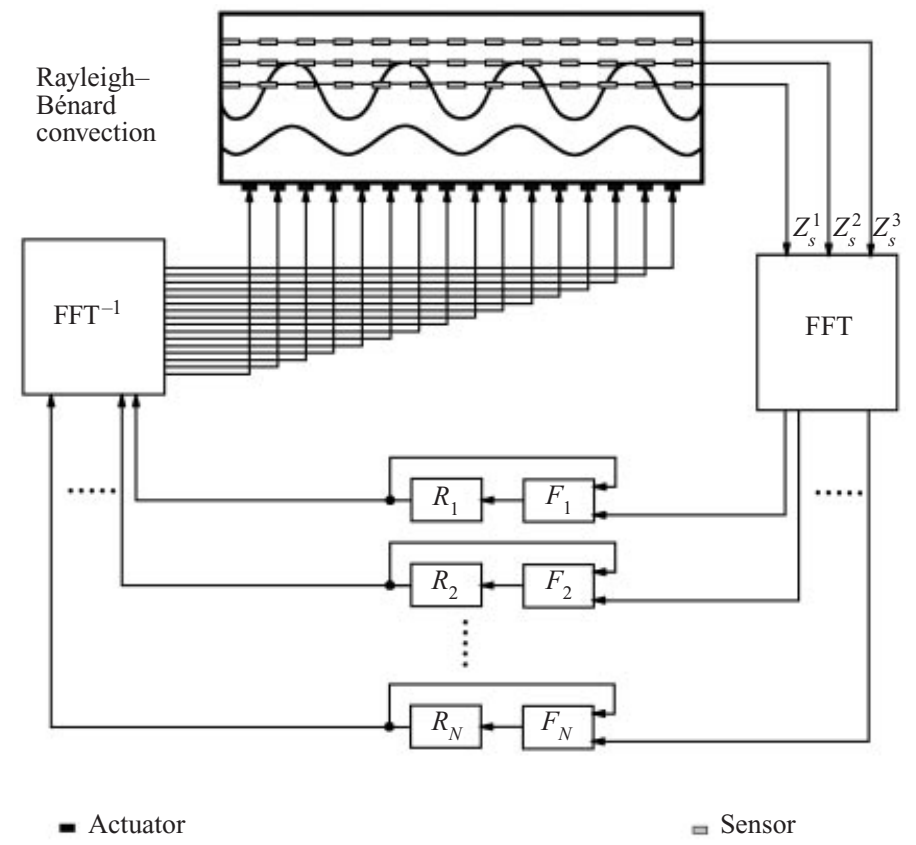

(b)

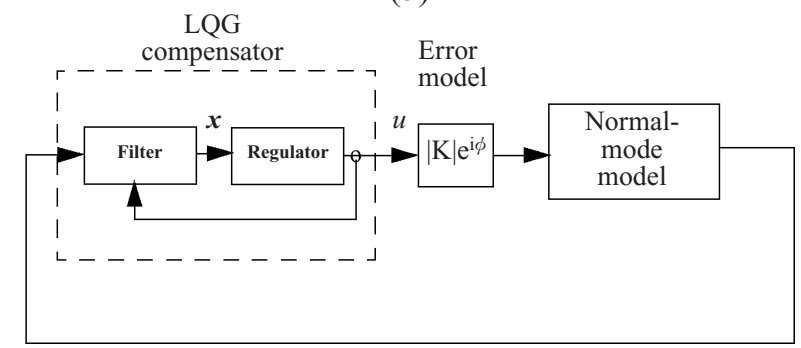

FIGURE 2. (a) Schematic for the physical implementation of the multi-wavenumber controller: $R_{n}$, regulator; $F_{n}$, filter; and FFT, fast Fourier transform. $(b)$ Block diagram of the control loop for a single wavenumber.

space. Sensors and actuators are interfaced to the controllers by fast Fourier transforms (FFT). Figure 2(a) links with simplicity the mathematical formulation to its computational implementation by summarizing in a block diagram the control strategy described above. The controllers can be programmed in a computer routine whose inputs are the arrays containing the temperatures measured by the planar sensors and whose output is an array containing the temperatures to be applied at the bottom the wall. The temperature measurements obtained by the planar sensors are converted by a fast Fourier transform into a set of modal sensor variables. Each pair of estimator and control blocks is integrated in time. Parallel computation produces the modal control variables. An inverse FFT converts the modal control variables into the actuating temperature at the bottom wall. This routine can be embedded in any Navier-Stokes solver able to handle time-dependent boundary conditions for the control of more realistic simulations of Rayleigh-Bénard convection.

Figure 2(a) also provides the basic architecture for the potential implementation 
of the present controller in an experiment and, eventually, in practical engineering applications. The temperature distribution at a given plane $\left(z_{s}^{(i)}\right)(i=1,2, \ldots, I)$ could be measured by a planar sensor constructed with an array of micro-electromechanical-system (MEMS) diode sensors (see Tang \& Bau 1998a,b). Analog to digital converters (A/D) and digital signal processors (DSP) would convert the measured temperatures $\boldsymbol{z}_{s}^{(i)}$ into the modal sensor variables. Each pair of estimator and control blocks would be replaced by a microprocessor, and a parallel computation produces the modal control variables. A DSP and a digital to analog converter (D/A) would produce the actuating signal. Finally, an array of MEMS heaters would provide the temperature distribution at the bottom wall (Tang \& Bau 1998a,b).

\subsection{State-space representation of temporal dynamics}

In this subsection, we consider a numerical procedure to represent the vertical dependence of the velocity and thermal field. We use the Galerkin approach (Gottlieb \& Orszag 1981) for the representation of the vertical dependence of the normal modes. The beam functions $\left\{\varphi_{m}\right\}$ are used as the basis functions for $W$ which has to satisfy four boundary conditions. On the other hand the sinusoidal functions $\left\{\beta_{m}\right\}$ are used as basis functions for $\Phi$ which only has to satisfy two boundary conditions. In our numerical computations, we truncate the infinite set to the first $N_{z}$ terms for both $W$ and $\Theta$. We use the same truncation number for both fields mainly for numerical convenience.

In general the Chebyshev polynomials have good convergence properties. However, in our application an individual polynomial does not satisfy the homogeneous boundary conditions. In contrast, individual beam functions do satisfy the homogeneous boundary conditions naturally and they converge reasonably fast for our stability analysis. Also, since we have transformed the thermal boundary condition at the lower wall to a homogeneous form, our results are not affected by Gibb's phenomenon in the vertical dependence. In practical applications when realistic lateral boundary conditions are incorporated, Gibb's phenomenon can affect the horizontal dependence (at small wavenumbers) and therefore the performance of the controller. However, the detrimental effects of Gibb's phenomenon can be substantially reduced using appropriate windowing techniques.

The Fourier coefficients for the vertical velocity $W(z, t)$ are expanded as follows:

$$
W(z, t)=\sum_{m=1}^{N_{z}} A_{m}(t) \varphi_{m}(z)=\sum_{m=1}^{N_{z}} A_{m}(t)\left[\frac{\sinh \left(a_{m} z+\mathrm{i} m \pi / 2\right)}{\sinh \left(a_{m}+\mathrm{i} m \pi / 2\right)}-\frac{\sin \left(a_{m} z+m \pi / 2\right)}{\sin \left(a_{m}+m \pi / 2\right)}\right] .
$$

Since the thermal boundary conditions for the planar sensor model and shadowgraph sensor model are different, the sinusoidal basis functions $\left\{\beta_{m}\right\}$ are different for the two cases. For the planar sensor model we let

$$
\Phi(z, t)=\sum_{m=1}^{N_{z}} B_{m}(t) \beta_{m}(z)=\sum_{m=1}^{N_{z}} B_{m}(t) \sqrt{2} \sin [m \pi(z+1 / 2)],
$$

and for the shadowgraph sensor model we have instead

$$
\Phi(z, t)=\sum_{m=1}^{N_{z}} B_{m}(t) \beta_{m}(z)=\sum_{m=1}^{N_{z}} B_{m}(t) \sqrt{2} \sin [(m-1 / 2) \pi(z-1 / 2)] .
$$


A convenient choice for $\Xi$ which satisfies the appropriate boundary conditions of the planar sensor case is

$$
\Xi(z, t)=(1 / 2-z) \Theta_{c}(t),
$$

and one which satisfies the boundary conditions of the shadowgraph sensor case is

$$
\Xi(z, t)=(z-1 / 2) Q_{c}(t) .
$$

For the planar case, we now substitute (2.30), (2.31) and (2.33) into (2.22) and (2.23), and perform the Galerkin projection. The following equations are obtained:

$$
\begin{gathered}
\operatorname{Pr}^{-1} \sum_{m=1}^{N_{z}}\left\langle\varphi_{j},\left(\partial_{z}^{2}-k^{2}\right) \varphi_{m}\right\rangle \dot{A}_{m}=\sum_{m=1}^{N_{z}}\left\langle\varphi_{j},\left(\partial_{z}^{2}-k^{2}\right)^{2} \varphi_{m}\right\rangle A_{m} \\
-k^{2} R a \sum_{m=1}^{N_{z}}\left\langle\varphi_{j}, \beta_{m}\right\rangle B_{m}-k^{2} R a\left\langle\varphi_{j},(1 / 2-z)\right\rangle \Theta_{c} \\
\dot{B}_{m}=\sum_{m=1}^{N_{z}}\left\langle\beta_{j},\left(\partial_{z}^{2}-k^{2}\right) \beta_{m}\right\rangle B_{m}+\sum_{m=1}^{N_{z}}\left\langle\beta_{j}, \varphi_{m}\right\rangle A_{m} \\
-\left\langle\beta_{j},(1 / 2-z)\right\rangle \dot{\Theta}_{c}+\left\langle\beta_{j},\left(\partial_{z}^{2}-k^{2}\right)(1 / 2-z)\right\rangle \Theta_{c}
\end{gathered}
$$

where the index $j$ runs from 1 to $N_{z}$ and the inner product $\langle$,$\rangle denotes integration$ over $z \in[-1 / 2,1 / 2]$. The corresponding equations for the shadowgraph model can be obtained from (2.35), (2.36) by replacing $\Theta_{c}$ by $Q_{c}$ and $(1 / 2-z)$ by $(z-1 / 2)$. Therefore the shadowgraph model equations will not be presented explicitly.

After substituting (2.31) and (2.33) into (2.27), we obtain $I$ measurement equations for the planar case,

$$
\Theta_{s}^{(i)}(t)=\sum_{m=1}^{N_{z}} B_{m}(t) \beta_{m}\left(z_{s}^{(i)}\right)+\left(1 / 2-z_{s}^{(i)}\right) \Theta_{c}, \quad i=1,2, \ldots, I .
$$

For the shadowgraph case, upon substitution of (2.32) and (2.34) into (2.29), we have a single measurement equation

$$
R_{s}(t)=\sum_{m=1}^{N_{z}} B_{m}(t) \int_{-1 / 2}^{1 / 2}\left(-k^{2}\right) \beta_{m}(z) \mathrm{d} z+Q_{c} \int_{-1 / 2}^{1 / 2}\left(-k^{2}\right)(z-1 / 2) \mathrm{d} z .
$$

As a final step, we construct a state vector $\boldsymbol{X}$ by arranging the coefficients $A_{m}$ and $B_{m}$ as follows:

$$
\boldsymbol{X} \equiv\left[A_{1}, A_{2}, \ldots, A_{N_{z}}, B_{1}, B_{2}, \ldots, B_{N_{z}}\right]^{\prime},
$$

where superscript ' denotes the transpose. Equations (2.35) and (2.36) can be rewritten in state-space form as

$$
\dot{\boldsymbol{X}}=\boldsymbol{A} \boldsymbol{X}+\boldsymbol{B}_{1} \boldsymbol{\Theta}_{c}+\boldsymbol{B}_{2} \dot{\boldsymbol{\Theta}}_{c},
$$

while the measurement equation (2.37) can be re-written as

$$
\boldsymbol{\Theta}_{s}=\boldsymbol{C} \boldsymbol{X}+\boldsymbol{D}_{3} \boldsymbol{\Theta}_{c} .
$$

In order to cast the matrix equation in a standard state-space form we can choose either $\boldsymbol{\Theta}_{c}$ or its time derivative as the control action variable. Here we define $\boldsymbol{u}=\boldsymbol{\Theta}_{c}$. The term $\dot{\boldsymbol{\Theta}}_{c}$ can be eliminated from (2.40) by defining a new state vector $\boldsymbol{x}=\boldsymbol{X}-\boldsymbol{B}_{2} \boldsymbol{\Theta}_{c}$. 
Upon transformation, the state-space equations can be written as

$$
\begin{aligned}
& \dot{x}=A x+B u, \\
& z=C x+D u,
\end{aligned}
$$

where $\boldsymbol{B}=\boldsymbol{B}_{1}+\boldsymbol{A} \boldsymbol{B}_{2}, \boldsymbol{D}=\boldsymbol{D}_{3}+\boldsymbol{C B}_{2}, \boldsymbol{u}=\boldsymbol{\Theta}_{c}$ and $\boldsymbol{z}=\boldsymbol{\Theta}_{s}$. Matrices $\boldsymbol{A}, \boldsymbol{B}$, and $\boldsymbol{C}$ contain the dynamics of the plant, actuators, and sensors, respectively. Matrix $\boldsymbol{D}$ contains the direct coupling between sensors and actuators.

The cost function of each wavenumber can be minimized individually, because of the orthogonality between pairs of Fourier modes. From (2.18), (2.19), following the normal decomposition, the cost function in state-space form for wavenumber $k$ is

$$
\mathscr{J}(k)=\int_{t}^{T}\left(\boldsymbol{z}^{\prime} \boldsymbol{z}+\gamma \boldsymbol{u}^{\prime} \boldsymbol{u}\right) \mathrm{d} \tau .
$$

In $\S 4$, we allow $z$ to be a vector but restrict $\boldsymbol{u}$ to a scalar quantity $u$.

\section{Optimal control theory}

In this section we describe the basic theory of the LQG control (Bryson \& Ho 1969), or, in modern terms, $\mathscr{H}_{2}$ control. A brief review will be given in a self-contained manner to provide the necessary governing equations for the closed-loop stability analysis.

The LQG problem is formulated as a stochastic optimal control problem described by equations

$$
\begin{gathered}
\dot{x}=A x+B u+\Gamma w, \\
z=C x+D u+v,
\end{gathered}
$$

where $\Gamma$ is an input matrix, $\boldsymbol{w}$ and $\boldsymbol{v}$ are both white noise processes with zero means and auto-correlation functions

$$
E\left[\boldsymbol{w}(t) \boldsymbol{w}^{\prime}(\tau)\right]=\boldsymbol{W} \delta(t-\tau), \quad E\left[\boldsymbol{v}(t) \boldsymbol{v}^{\prime}(\tau)\right]=\boldsymbol{V} \delta(t-\tau),
$$

where $E[\cdot]$ is the expectation operator averaging over all underlying random variables and $\delta(t-\tau)$ is the delta function. Note that $\boldsymbol{W}$ and $\boldsymbol{V}$, the power spectral densities, will be chosen here as design parameters to enhance system performance. In our case $\boldsymbol{\Gamma}$ will be taken as $\boldsymbol{B}$, implying that the disturbances, in a manner similar to the control, enter the system dynamics at the wall.

The LQG controller is determined by finding the control action $\boldsymbol{u}\left(Z_{t}\right)$, where $Z_{t}=\{z(\tau) ; 0 \leqslant \tau \leqslant t\}$ is the measurement history, which minimizes the cost criterion

$$
J=\lim _{T \rightarrow \infty} \frac{1}{T-t} E\left[\int_{t}^{T}\left(\boldsymbol{x}^{\prime} \mathbf{Q} \boldsymbol{x}+2 \boldsymbol{x}^{\prime} \mathbf{N} \boldsymbol{u}+\boldsymbol{u}^{\prime} \mathbf{R} \boldsymbol{u}\right) \mathrm{d} \tau\right]
$$

subject to the stochastic dynamic system model equations (3.1) and (3.2). The division by $(T-t)$ ensures that the cost criterion remains finite in the presence of uncertainties in the infinite-time problem $(T \rightarrow \infty)$. Note that (3.4) can include (2.44) where

$$
J=\lim _{T \rightarrow \infty} \frac{1}{T-t} E[\mathscr{J}(k)] .
$$

Note also that even though the time interval is infinite, time response is still measured by the eigenvalues of the closed-loop system. We consider the infinite-time problem with a time-invariant dynamics system because the controller gains become constants. 
For $\boldsymbol{Q}$ and $\boldsymbol{N}$ chosen to be consistent with the cost criterion (2.44) (see (3.18)), the cost criterion will remain positive definite (see Bryson \& Ho (1969) for necessary and sufficient conditions for optimality with general $\boldsymbol{Q}$ and $\boldsymbol{N}$ ).

By nesting the conditional expectation with respect to $Z_{t}$ within the unconditional expectation of (3.4), i.e. $E[\mathscr{J}(k)]=E\left[E\left[\mathscr{J}(k) / Z_{t}\right]\right]$ where $E\left[\cdot / Z_{t}\right]$ denotes the expectation $(\cdot)$ conditioned on $Z_{t}$, the cost criterion can be written as

$$
J=\lim _{T \rightarrow \infty} \frac{1}{T-t} E\left[\int_{t}^{T}\left[\hat{\boldsymbol{x}}^{\prime} \mathbf{Q} \hat{\boldsymbol{x}}+2 \hat{\boldsymbol{x}}^{\prime} \boldsymbol{N} \boldsymbol{u}+\boldsymbol{u}^{\prime} \mathbf{R} \boldsymbol{u}+\operatorname{tr}(\boldsymbol{P})\right] \mathrm{d} \tau\right],
$$

where $\hat{\boldsymbol{x}}=E\left[\boldsymbol{x} / Z_{t}\right]$ is the conditional mean estimate of the state $\boldsymbol{x}$. The term $\operatorname{tr}(\boldsymbol{P})$ is the trace of the error variance matrix which naturally occurs as a result of taking the conditional expectation into the integrand of the cost criterion. This cost criterion is now minimized subject to the estimation equations discussed below. Note that $\boldsymbol{P}$ does not depend on the control (see (3.9) below) and therefore does not enter into the optimization process.

The solution to the regulator problem (Bryson \& Ho 1969) is a compensator composed of a state reconstruction process, known here as a filter (in the no-noise case it is known as an observer) in cascade with a controller (see figure $2 b$ ). The state estimate (conditional mean) is called the Kalman filter, and is governed by

$$
\dot{\hat{\boldsymbol{x}}}=\boldsymbol{A}^{*} \hat{\boldsymbol{x}}+\boldsymbol{B}^{*} \boldsymbol{u}+\boldsymbol{K}_{f} \boldsymbol{v}, \quad \boldsymbol{v}=z-\hat{z}=\boldsymbol{C}^{*}(\boldsymbol{x}-\hat{\boldsymbol{x}})+\boldsymbol{v},
$$

where the matrices with asterisk superscripts correspond to the nominal point $\left(k^{*}, R a^{*}\right)$. The Kalman gain matrix $\boldsymbol{K}_{f}$, constructed to trade the accuracy of the new measurements against the accuracy of the state propagated from the system dynamics, is given by

$$
\boldsymbol{K}_{f}=\boldsymbol{P} \mathbf{C}^{* \prime} \boldsymbol{V}^{-1},
$$

where $\boldsymbol{P}$ is the error variance in the statistical problem. In the infinite-time stationary formulation, the error variance $\boldsymbol{P}$ is the solution to the algebraic Riccati equation (ARE),

$$
\boldsymbol{A}^{*} \boldsymbol{P}+\boldsymbol{P} \boldsymbol{A}^{* \prime}+\boldsymbol{\Gamma} \boldsymbol{W} \boldsymbol{\Gamma}^{\prime}-P \boldsymbol{C}^{* \prime} \boldsymbol{V}^{-1} \boldsymbol{C}^{*} \boldsymbol{P}=\mathbf{0} .
$$

If the system is $\left(\boldsymbol{A}^{*}, \boldsymbol{C}^{*}\right)$ observable and $\left(\boldsymbol{A}^{*}, \boldsymbol{\Gamma}\right)$ controllable, then $\boldsymbol{P}$ is positive definite. Under these assumptions, it can be shown that the difference between the internal state $\boldsymbol{x}$ and the estimated state $\hat{\boldsymbol{x}}$, i.e. the error

$$
\boldsymbol{e}=\boldsymbol{x}-\hat{\boldsymbol{x}}
$$

goes to zero as time goes to infinity. In other words, the evolution equation

$$
\dot{e}=A_{f} e+K_{f} v+\Gamma w,
$$

is stable, i.e. all the eigenvalues of the matrix

$$
\boldsymbol{A}_{f}=\boldsymbol{A}^{*}-\boldsymbol{K}_{f} \mathbf{C}^{*}
$$

have negative real part.

Minimizing the infinite-time cost function $J$, (3.6) subject to (3.7) yields the following control law:

where

$$
\boldsymbol{u}=-\boldsymbol{K}_{c} \hat{\boldsymbol{x}}
$$

$$
\boldsymbol{K}_{c}=\boldsymbol{R}^{-1}\left(\boldsymbol{B}^{* \prime} \boldsymbol{S}+\boldsymbol{N}^{\prime}\right),
$$


and $\boldsymbol{S}$ is the solution of the algebraic Riccati equation (ARE)

$$
\boldsymbol{A}^{* \prime} \boldsymbol{S}+\boldsymbol{S} \boldsymbol{A}^{*}+\boldsymbol{Q}-\left(\boldsymbol{S} \boldsymbol{B}^{*}+\boldsymbol{N}\right) \boldsymbol{R}^{-1}\left(\boldsymbol{B}^{* \prime} \boldsymbol{S}+\boldsymbol{N}^{\prime}\right)=\mathbf{0} .
$$

It should be remarked that the control gain matrix $\boldsymbol{K}_{c}$ is determined from functions only of the known dynamic coefficients $\left(\boldsymbol{A}^{*}, \boldsymbol{B}^{*}\right)$ and weightings in the cost criterion $(\boldsymbol{Q}, \boldsymbol{R})$, and not the statistics of the input $(\boldsymbol{V}, \boldsymbol{W})$. Consequently, $\boldsymbol{K}_{c}$ is determined from a performance index such as (3.4), independent of the stochastic inputs. If $\left(\boldsymbol{A}^{*}, \boldsymbol{B}^{*}\right)$ is controllable and $\left(\boldsymbol{A}^{*}, \boldsymbol{Q}^{1 / 2}\right)$ observable, then the loop coefficient matrix

$$
\boldsymbol{A}_{c}=\boldsymbol{A}^{*}-\boldsymbol{K}_{c} \boldsymbol{B}^{*}
$$

is stable. The controllable and observable conditions can be weakened to stabilizable and detectable (Kwakernaak \& Sivan 1972).

When we combine the estimator and the regulator, the dynamic system composed of the controlled process and filter becomes

$$
\left(\begin{array}{c}
\dot{\boldsymbol{e}} \\
\hat{\boldsymbol{x}}
\end{array}\right)=\left[\begin{array}{cc}
\boldsymbol{A}_{f} & \mathbf{0} \\
\boldsymbol{K}_{f} \boldsymbol{C}^{*} & \boldsymbol{A}_{c}
\end{array}\right]\left(\begin{array}{c}
\boldsymbol{e} \\
\hat{\boldsymbol{x}}
\end{array}\right)+\left(\begin{array}{c}
\boldsymbol{K}_{f} \boldsymbol{v}+\boldsymbol{\Gamma} \boldsymbol{w} \\
\boldsymbol{K}_{f} \boldsymbol{v}
\end{array}\right) .
$$

Note that any choice of two among $\boldsymbol{e}, \hat{\boldsymbol{x}}$ and $\boldsymbol{x}$ produces the same dynamics because they are algebraically related by (3.10). Under the above controllability and observability assumptions, $\boldsymbol{A}_{f}$ and $\boldsymbol{A}_{c}$ have only stable eigenvalues if optimal gains $\boldsymbol{K}_{f}$ and $\boldsymbol{K}_{c}$ of (3.12), (3.16) are used. Other schemes such as $\mathscr{H}_{\infty}$ could be proposed (Rhee \& Speyer 1991), but from experience these schemes seem to produce only secondary modifications to the system performance over our LQG controller.

The infinite-time stationary formulation will be used in our study. The LQG control loop is shown in the block diagram of figure $2(b)$. Note that the cost function (2.44) can be expressed in the standard form (3.4), if we let

$$
\boldsymbol{Q}=\boldsymbol{C}^{* \prime} \boldsymbol{C}^{*}, \quad \boldsymbol{N}=\boldsymbol{C}^{* \prime} \boldsymbol{D}^{*}, \quad \boldsymbol{R}=\left(\gamma \boldsymbol{I}+\boldsymbol{D}^{* \prime} \boldsymbol{D}^{*}\right) .
$$

Since the power spectral density is not known, for simplicity of the design we consider $\boldsymbol{V}$ and $\boldsymbol{W}$ to be of the form $\boldsymbol{V}=\alpha \boldsymbol{l}$ and $\boldsymbol{W}=\beta \boldsymbol{I}$ where $\alpha$ and $\beta$ are scalar and $\boldsymbol{I}$ is a unity matrix. Only the ratio of $\alpha$ with $\beta$ is important.

The process noise spectral density $\beta$ and the weighting $\gamma$ in the cost function are considered design parameters. The case where $\gamma \rightarrow 0$ corresponds to unlimited control authority of the full-state feedback controller. The choice $\boldsymbol{\Gamma}=\boldsymbol{B}^{*}$ allows for looptransfer recovery (Doyle \& Stein 1979). Loop-transfer recovery of the LQG controller to full-state feedback guarantees that robust performance occurs when the process noise goes to infinity, i.e. $\beta \rightarrow \infty$, provided there exists no non-minimal-phase zero in the plant. In our case, there are non-minimal-phase zeros, but a partial recovery is still shown to be possible (Turan, Mingori \& Goodwin 1994).

As we have noted in $\S 2$, the analysis will be based on a single normal-mode model because the normal modes decouple. Although only one controller is needed at $\left(R a^{*}, k^{*}\right)$, it is implemented for different $k^{*}$ over a range of wavenumbers. The design point is determined so that when the controller is implemented, no unstable region appears below the neutral curve. Although the plant has multiple outputs, the system can be analysed in terms of robustness as a single-input/single-output (SISO) system by breaking the loop at the plant input (see figure $2 b$ ). We denote the output $u$ of the controller by $u_{0}$ and the input $u$ to the plant by $u_{i}$. The open-loop system of equations formed by breaking the loop at the input to the plant is

$$
\dot{\boldsymbol{x}}_{a}=\boldsymbol{A}_{a} \boldsymbol{x}_{a}+\boldsymbol{B}_{a} u_{i}, \quad u_{0}=\boldsymbol{C}_{a} \boldsymbol{x}_{a}+\boldsymbol{D}_{a} u_{i},
$$


where the augmented state composed of the plant and compensator in cascade is $\boldsymbol{x}_{a}=\left[\boldsymbol{x}^{\prime}, \hat{\boldsymbol{x}}^{\prime}\right]^{\prime}$. The coefficient matrices are given by

$$
\left.\begin{array}{c}
\boldsymbol{A}_{a}=\left[\begin{array}{cc}
\boldsymbol{A} & \mathbf{0} \\
\boldsymbol{K}_{f} \boldsymbol{C} & \left(\boldsymbol{A}^{*}-\boldsymbol{B}^{*} \boldsymbol{K}_{c}-\boldsymbol{K}_{f} \boldsymbol{C}^{*}+\boldsymbol{K}_{f} \boldsymbol{D}^{*} \boldsymbol{K}_{c}\right)
\end{array}\right], \quad \boldsymbol{B}_{a}=\left[\begin{array}{c}
\boldsymbol{B} \\
\boldsymbol{K}_{f} \boldsymbol{D}
\end{array}\right], \\
\boldsymbol{C}_{a}=\left[\mathbf{0},-\boldsymbol{K}_{c}\right], \quad \boldsymbol{D}_{a}=\mathbf{0} .
\end{array}\right\}
$$

The evolution equation for the closed-loop feedback system is

$$
\left(\begin{array}{c}
\dot{\boldsymbol{x}} \\
\hat{\boldsymbol{x}}
\end{array}\right)=\left[\begin{array}{cc}
\boldsymbol{A} & -K \boldsymbol{B} \boldsymbol{K}_{c} \\
\boldsymbol{K}_{f} \boldsymbol{C} & \left(\boldsymbol{A}^{*}-\boldsymbol{K}_{f} \boldsymbol{C}^{*}-\boldsymbol{B}^{*} \boldsymbol{K}_{c}+\boldsymbol{K}_{f}\left(\boldsymbol{D}^{*}-K \boldsymbol{D}\right) \boldsymbol{K}_{c}\right)
\end{array}\right]\left(\begin{array}{c}
\boldsymbol{x} \\
\hat{\boldsymbol{x}}
\end{array}\right),
$$

where $u_{i}=K u_{0}$. In the above equation, matrices with an asterisk superscript correspond to the design parameters $k^{*}$ and $R a^{*}$. Note that in the particular case when the plant operates at nominal design condition, i.e. $k=k^{*}, R a=R a^{*}$ and $K=1$, the closed-loop poles will correspond to the ensemble of eigenvalues of $\boldsymbol{A}_{f}$ and of $\boldsymbol{A}_{c}$. In other words, (3.21) reduces to (3.17) and the filter poles and regulator poles decouple. One can show this from the transformation

$$
\left(\begin{array}{l}
x \\
\hat{x}
\end{array}\right)=\left[\begin{array}{ll}
I & I \\
0 & I
\end{array}\right]\left(\begin{array}{l}
e \\
\hat{x}
\end{array}\right),
$$

where $\boldsymbol{I}$ is an identity matrix.

In general, the plant does not operate at the nominal design condition. Consequently, there is a mismatch between the parameters $\left(k^{*}, R a^{*}\right)$ used to design the controller and the operating parameters $(k, R a)$. Our analysis uses two methods to characterize the robustness of the stabilized system: neutral curves and gain and phase margins. In the first method, we select the nominal points $\left(k^{*}, R a^{*}\right)$ and construct the region of stability of the dynamics system (3.21) as $R a$ and $k$ vary with $K=1$. The boundary of this region is where the real part of the least-stable closed-loop pole of (3.21) becomes zero. This boundary curve in the $(k, R a)$-plane is called the neutral curve. We identify the minimum of $R a$ with respect to wavenumber on the neutral curve, so that the range of $R a$ from zero to this minimum, along with the whole range of wavenumbers, is stable. By robustness we mean constructing the largest range of $R a$ from zero up to this minimum.

The second method used to estimate robustness is the classical gain and phase margins approach. This approach allows us to characterize robustness with respect to more general uncertainties, such as unmodelled dynamics. To obtain the gain and phase margins, we consider an error model $K=|K| e^{i \phi}$ (see figure $2 b$ ), with the plant operating at the nominal parameters $k=k^{*}$ and $R a=R a^{*}$. The shifts of $|K|$ and $\phi$ from these nominal values $(|K|=1, \phi=0)$ to where the system becomes unstable are essentially the gain and phase margins, respectively. Their values can be determined from accompanying Nyquist plots. The gain and phase margins are defined explicitly in $\S 4.2 .1$ where these values indicate the amount of gain and phase change that the system can tolerate due to uncertainties in the system dynamics.

\section{Results}

In this paper, we consider the condition of $\mathrm{Pr}=7$ which enables us to compare our numerical results with those of Tang \& Bau (1994, 1998b) and Howle (1997a). In their experiments, however, Howle $(1997 b, c)$ used a high- $P r$ fluid $(\operatorname{Pr} \approx 200)$ whereas 
in Tang \& Bau (1998a) the Pr value of their testing fluid was not given. It should be noted that while the stability properties in the uncontrolled case are independent of $\mathrm{Pr}$, they are $\mathrm{Pr}$ dependent in the controlled case.

Our numerical results have been obtained using $N_{z}=26$ (see § 2.4 ) which appears to be adequate for our stability analysis. For example, consider the closed-loop eigenvalue problem of (3.21) with controller design values $R a^{*}=14.8 R a_{c 0}$ and $k^{*}=3.15$ and the system evaluated at $k=5.5$ and $R a=14.52$ which lies on the neutral curve (see figure $8(d)$ ), the norm of eigenvector (square root of the mean-square sum of entries) of the neutral eigenvalue of the coefficient matrix of (3.21) appears well converged. When $N_{z}=26$ is increased to 52 , this norm changes only by less than $0.7 \%$.

\subsection{Proportional feedback control}

For convection in a layer of fluid bounded by rigid walls with prescribed temperatures, it is well known that the critical Rayleigh number $R a_{c 0}=1707.76$ occurs at wavenumber $k_{c 0}=3.117$ (Chandrasekhar 1961). Instead, when heat flux is prescribed at the lower wall, the critical value $R a_{c 0} \approx 1295.78$ occurs at $k_{c}=2.552$.

In the case of proportional feedback control, the control law is $u=-K_{p} z$, where $K_{p}$ is a constant proportional gain. We consider the planar sensor model to illustrate the effects of feedback control upon stability, and the results are shown in figure $3(a)$.

In this figure three neutral curves are shown: each curve consists of a heavy and a thin solid line, representing a monotonic mode and an oscillatory mode of convection, respectively. The oscillatory mode corresponds to a complex conjugate pair of eigenvalues. The three curves correspond to three sensor locations: $z_{s}=0$ at the mid-plane and $z_{s}= \pm 0.1$. The offset with respect to the mid-plane is $10 \%$ of the thickness of the fluid layer and gives a substantial shift in stability properties. The unstable and stable regions are separated by a neutral curve and are identified by the letters $\mathrm{U}$ and $\mathrm{S}$, respectively. In each case the maximum $R a$ achievable corresponds to the crossing point between the heavy and thin line.

For $z_{s}=0$, the monotonic mode is the lowest even mode of convection since the first odd mode is unobservable. In fact, in this case the sensor plane coincides with the node of the first odd mode. As $K_{p}$ increases beyond the crossing point the critical $R a$ decreases. With this in mind, a pole-zero map and a root locus diagram are helpful to understand the stability behaviour. Figure $3(b)$ shows the open-loop poles $(\times)$ and zeros (O) and figure 3(c) the corresponding root locus diagram. For a given $R a$, as $K_{p}$ increases from zero the unstable pole moves to the left while a stable pole moves to the right. Subsequently, the two poles coalesce. After coalescence a pair of complex conjugate poles (corresponding to the oscillatory mode) break off the real axis. The break-away point (where the coalescence occurs) moves to the right as $R a$ increases. The crossing point in figure $3(a)$ corresponds to the coalescence at the origin in figure $3(c)$. As $R a$ increases and keeping $K_{p}$ constant, the closed-loop poles move to the right.

From the root locus of figure 3(c), the results of figure 3(a) can be interpreted as follows. For $R a$ above the crossing point, the system is unstable for any gain $K_{p}$. For $R a$ below the crossing point, there exists a finite range of gain $K_{p}$ in which the system is stable. The lower end point of the range corresponds to the minimal value of $K_{p}$ required in order to move the monotonic pole to left-half $s$-plane. The upper end point of the range corresponds to the maximal value of $K_{p}$ that can be used before the pair of complex conjugate modes become unstable.

The stability diagram for the shadowgraph sensor model can be found in Howle 

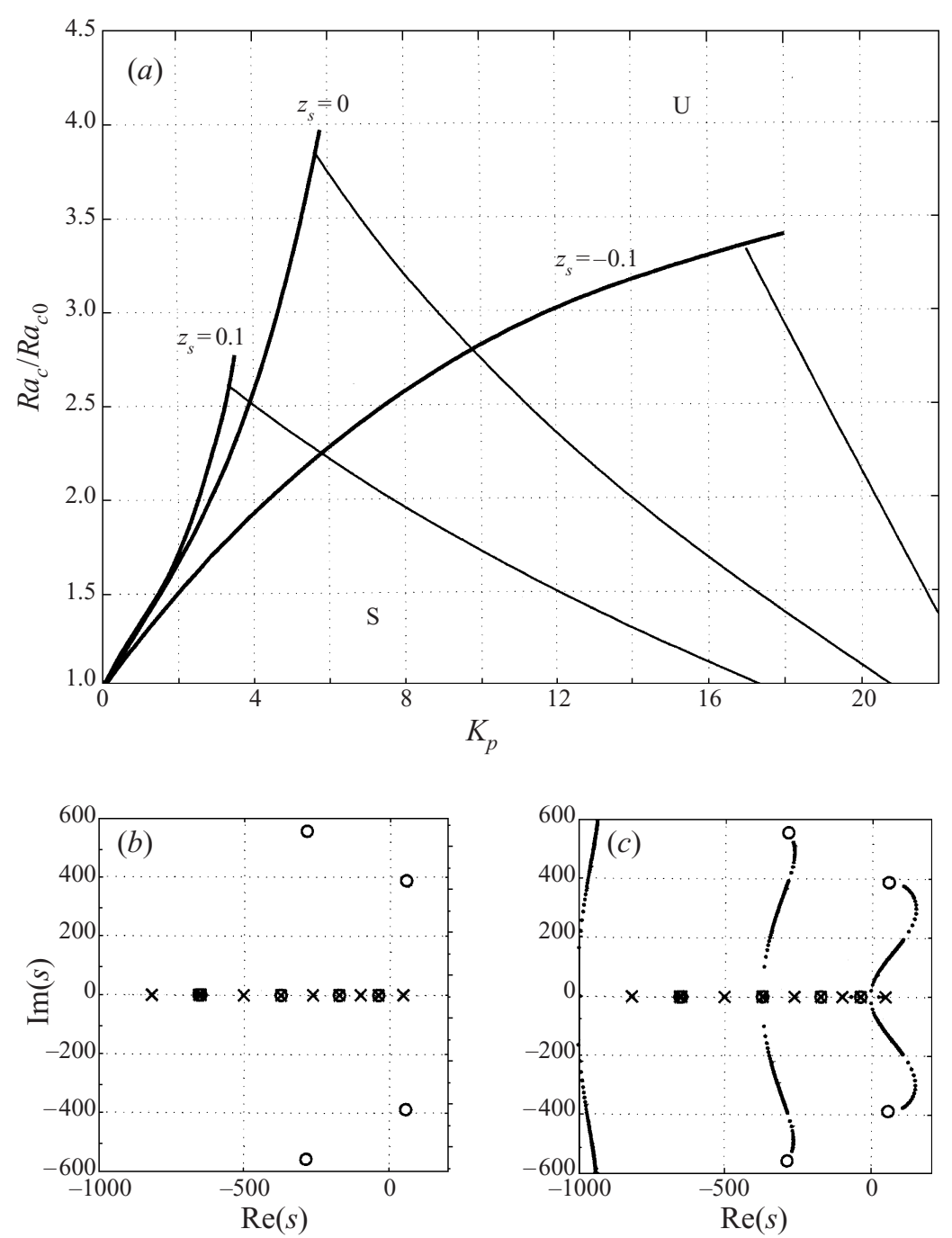

FIGURE 3. (a) Neutral curves for the planar sensor model using the proportional feedback control, corresponding to $z_{s}=-0.1,0$ and 0.1 (heavy and thin lines indicate monotonic and oscillatory onset, respectively). (b) Pole-zero diagram of the plant for $k=4.4, R a=3.5 R a_{c 0}$ and (c) corresponding root loci for $K_{p}$ varied between 0 and 2000.

(1997a). In this case there are no unstable complex conjugate modes. As $K_{p}$ increases to $\infty$, the critical $R a$ increases monotonically to about 3.13 times $R a_{c 0}$.

\section{2. $L Q G\left(\mathscr{H}_{2}\right)$ control}

The limitation in the performance of proportional feedback control provides the motivation for developing LQG controllers. We will apply the LQG synthesis method to both planar sensor and shadowgraph sensor models. We first seek to reduce the number of design parameters in our analysis. For a given set of physical parameters we examine the closed-loop eigenvalues and observe that for a stable system the real part of the least-stable eigenvalue has its largest magnitude when $\gamma \rightarrow 0$ and $\beta \rightarrow \infty$. Since the observed improvements become less significant for $\gamma<0.1$ and $\beta>100$, we let $\gamma=0.1$ and $\beta=100$. 

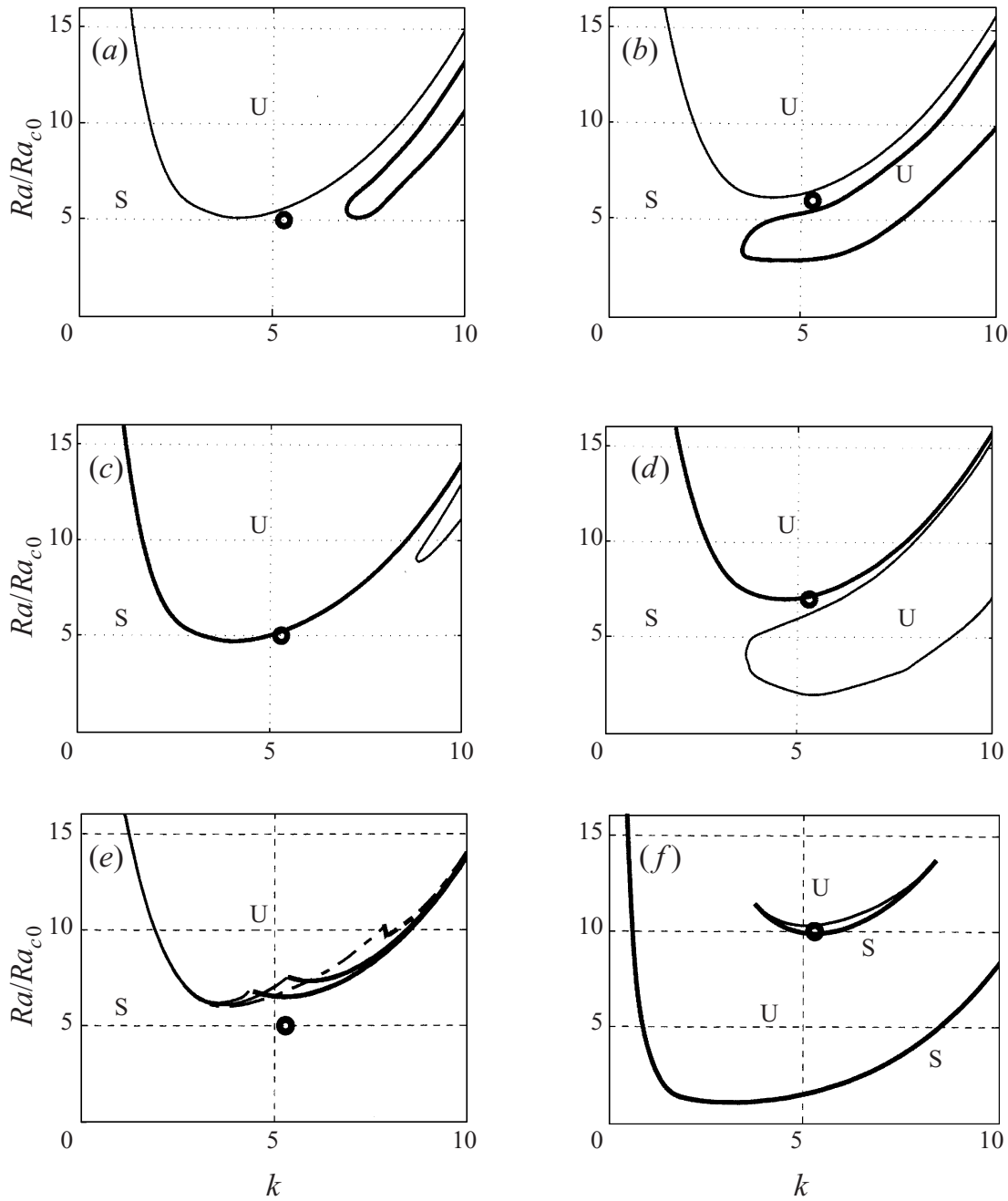

FIGURE 4. Stability diagrams for the planar sensor model with one sensor, using the LQG control. The nominal condition $\left(k^{*}, R a^{*}\right)$ is denoted by a solid circle. The heavy and thin lines correspond to the monotonic and oscillatory onsets, respectively. $(a, b, f) z_{s}=0.15$ and $(c, d) z_{s}=-0.15$. $(a-e)$ $R a^{*}=5 R a_{c 0}$ and $k^{*}=5.3$ and $(f) R a^{*}=10 R a_{c 0}$ and the same $k^{*}$. In $(e)$ the sensitivity about $z_{s}=0$ with respect to $z_{s}$ is shown. In $(f)$ a vast lower unstable region developed at a sufficiently high $R a^{*}$ is shown.

\subsubsection{Planar sensor model}

(i) One-sensor control

The one-sensor model is especially convenient for understanding the closed-loop stability properties of the system. Once the qualitative properties of this case are understood, the performance of the controller will be improved by adding additional sensors.

Figure $4(a-f)$ shows the neutral stability diagram in the $(k, R a)$-plane for a controller designed at the nominal values $k^{*}$ and $R a^{*}$. The nominal point $\left(k^{*}, R a^{*}\right)$ is indicated by a solid circle in the figures. The thin line and heavy line curves correspond to the neutrally stable oscillatory and monotonic modes, respectively. In figure $4(a, b)$ the sensor plane is located at $z_{s}=0.15$. We use a larger sensor displacement with 
respect to the mid-plane than the one used for the proportional feedback control to emphasize the effect on the neutral curve. Figure $4(a)$ shows the neutral curves for a controller designed at the nominal point $R a^{*}=5 R a_{c 0}$ and $k^{*}=5.3$. The neutral curves have two minima, and the value $k^{*}=5.3$ has been chosen to make the minima nearly the same. The controller stabilizes the system for any $R a<R a^{*}$. To characterize the stability of the controlled system with respect to $R a^{*}$, we re-design the controller for $R a^{*}=6 R a_{c 0}$ while maintaining $k^{*}$ fixed. Figure $4(b)$ shows a dramatic change in the neutral curve: the banana-shaped branch moves downward giving rise to an unstable region below $R a^{*}$.

To further characterize the stability of the controlled system with respect to the location of the sensor, we move the sensor plane at $z_{s}=-0.15$. We design a controller for $k^{*}=5.3$, as before, and $R a^{*}=5 R a_{c 0}$ (figure $4 c$ ) and $R a^{*}=7 R a_{c 0}$ (figure $4(d)$ ). Figures $4(c)$ and $4(d)$ show similar stability characteristics as those in figures $4(a)$ and $4(b)$. However, the two branches of the neutral stability curves switch roles. The left branch now represents the monotonic onset while the right branch represents the oscillatory onset.

The role switch in relation to the location of the sensor plane deserves a closer examination. We consider a smaller perturbation of the sensor location with respect to the mid-plane. Figure 4(e) shows the stability limits for three very close sensor locations. At $z_{s}=0$ (solid lines), the neutral curve is composed of an oscillatory segment on the left and a monotonic segment on the right. At $z_{s}=0.005$ (dasheddotted lines), the monotonic segment of the neutral curve retreats rightward while the oscillatory segment dominates the range. At $z_{s}=-0.005$ (dashed lines), the opposite effect occurs: the oscillatory segment of the neutral curve retreats to the left. These results are consistent with the results presented in figures $4(a-d)$. Since significant distortions and shifts of the stability limits have occurred within a very small perturbation of $z_{s}$, sensitivity to sensor location becomes an important factor for the practical implementation of the sensors. As subsequent results will show, this type of sensitivity is no longer present if three sensors are incorporated.

In order to show that the lower unstable region will become much larger with further increase of $R a^{*}$, we design a controller at the nominal values $R a^{*}=10 R a_{c 0}$ and $k^{*}=5.3$ and place the sensor plane at $z_{s}=0.15$ for an illustration. Figure $4(f)$ shows a thin island of stability in the unstable region. This stable region is bounded by two neutral curves which coalesce on the right and intersect on the left. Figure $4(a-f)$ reveals the occurrence of an unstable region at $R a<R a^{*}$ which severely restricts the achievable degree of stabilization.

The results of proportional feedback control have demonstrated the significance of the sensor location at $z_{s}=0$. This location gives the maximum range of stabilization even for the LQG controller. We observe that $R a^{*}$ can be raised to $10 R a_{c 0}$ at properly chosen values of $k^{*}$ (see figure $5 a-c$ ) without inducing a large lower unstable region, if the sensor is placed at the mid-plane. At this $R a^{*}$, the system is stable up to the critical $R a$ of the first odd mode $\left(R a_{c} \approx 10.31 R a_{c 0}\right.$ and $k_{c}=5.36$, see Chandrasekhar 1961), since the first odd mode is not stabilizable because it is unobservable.

Hence, there is no reason to place $R a^{*}$ above $10.31 R a_{c 0}$. Below this value, however, the critical point of the neutral curve lies to the right of the nominal point if $k^{*}$ is sufficiently small, or to the left of the nominal point if $k^{*}$ is sufficiently large. For this case, we can use two nominal points to lift the overall neutral curve to coincide with the neutral curve of the first odd mode.

Consider $R a^{*}=10 R a_{c 0}$, just below the $R a_{c}$ of the first odd mode. We choose the two nominal $k^{*}$ on both sides of $k_{c}=5.36$. The values $k^{*}=4$ and 6 (marked by solid 

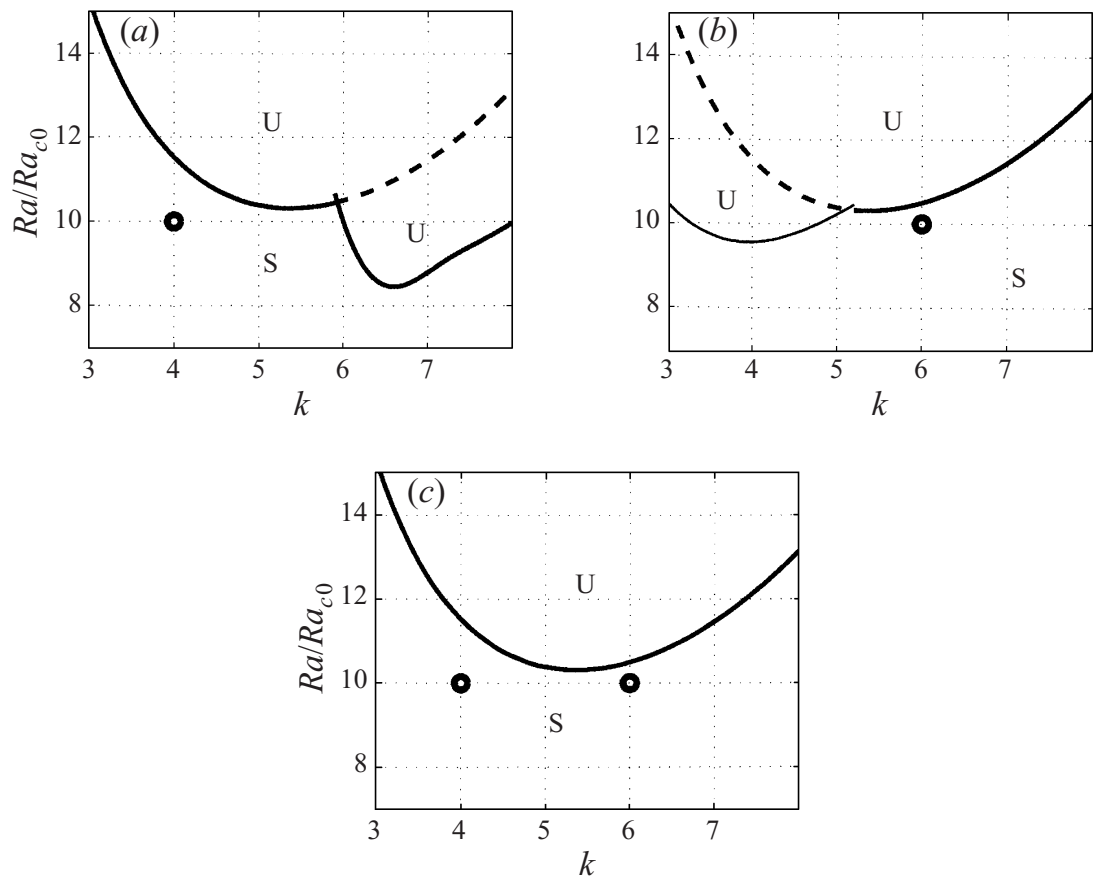

FIGURE 5. Stability diagrams for the planar sensor model with a single mid-plane sensor: $(a)$ neutral curve for the nominal condition $k^{*}=4$ and $R a^{*}=10 R a_{c 0} ;(b)$ neutral curve for the nominal conditions $k^{*}=6$ and $R a^{*}=10 R a_{c 0} ;(c)$ the resulting neutral curve by incorporating the two sets of nominal conditions. This curve coincides with the neutral curve of the first odd mode of convection in the uncontrolled case.

circles) produce small dips in the unstable region and are reasonable to use as nominal points. Figure $5(a-c)$ illustrates how the stability limit is determined by the principle of superposition. The nominal point at $k^{*}=4$ in figure $5(a)$ corresponds to the unstable region delimited by the heavy solid lines which has a minimum $R a \approx 8.4 R a_{c 0}$. For $k<5.9$, the stability limit corresponds to the neutral curve of the first odd mode. Similarly, in figure $5(b)$ the second nominal point at $k^{*}=6$ corresponds to the unstable region delimited by the thin and heavy solid lines which has a minimum at about $9.5 R a_{c 0}$. The thin curve corresponds to an oscillatory onset. The heavy curve coincides with a segment of the neutral curve of the odd mode. If both nominal points are used for the controllers, then the overall stability limit coincides with the neutral curve of the first odd mode upon superposition. The controllers designed at the first nominal point $k^{*}=4$ operate over the band $0<k<k_{c} \approx 5.36$, while the controller designed at $k^{*}=6$ operates over the wavenumbers greater than $k_{c}$.

The result shows that the degree of stabilization is significantly higher than that achievable with the proportional control. Unfortunately, the one-sensor design is not sufficiently robust with respect to the location of the sensor plane. This problem is significant because a perfect sensor placement is not achievable in practice. To demonstrate the sensitivity, in figure $6(a)$ we consider a planar sensor at $z_{s 1}=0.01$, i.e. slightly off the mid-plane, and re-design the controller for $R a^{*}=10 R a_{c 0}$ and $k^{*}=4$. Figure 6(a) shows the presence of a thin stable region in the middle of the unstable region. This stable region is bounded from above by a neutral curve of an oscillatory mode (thin line) and below by a neutral curve of a monotonic mode (heavy solid 

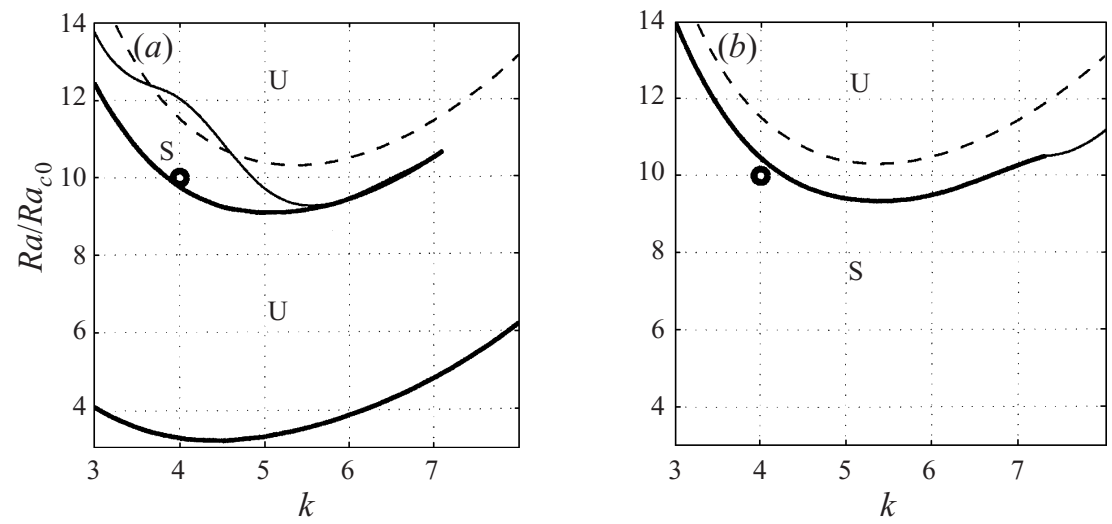

FIGURE 6. (a) Stability diagram for the one-sensor model with a small offset $z_{s}=0.01$. Results show a vast lower unstable region below $R a^{*}=10 R a_{c 0}$. The heavy (thin) solid line corresponds to a monotonic (oscillatory) onset. (b) Stability diagram for the two-sensor model, with a second sensor positioned at $z_{s}=-0.01$. As a result, the lower unstable region is eliminated.

line). The stable region exists for $k<3$. As $k$ increases it becomes a very narrow strip which eventually terminates at $k \approx 7.1$, similar to the behaviour in figure $4(f)$. For comparison the neutral curve for the $z_{s}=0$ case (thin dashed line) is also included in figure 6(a). Comparing figure 6(a) to figure 5(c), we see a dramatic difference in stability properties due to a small shift of sensor location of 0.01 . Fortunately, this sensitivity can be significantly reduced by introducing a second sensor located close to the mid-plane. Figure $6(b)$ shows the stability diagram when a second sensor is included. This case will be discussed in the next subsection.

To characterize the robustness of the controlled system with respect to plant uncertainties we compute gain and phase margins at $R a=R a^{*}$ and $k=k^{*}$. In all the cases considered, the open-loop system has one unstable pole so that for closedloop stability the Nyquist locus encircles (counter-clockwise) the point $(-1,0)$ once. Because of this property, in general there exists an upper and lower value for each gain and phase margin. The upper and lower gain margins are designed to measure how much the gain $K$ can be decreased, or increased, before the system becomes unstable (figure $2 b$ ). Likewise, the upper and lower phase margins are designed to measure how much the phase can be decreased, or increased, before the system becomes unstable. Accordingly, the lower and upper gain margins are defined as $20 \log _{10}\left(1 / X_{1}\right)(\mathrm{dB})$ and $20 \log _{10}\left(X_{2}\right)(\mathrm{dB})$, respectively, where $X_{1}\left(\left|X_{1}\right|<1\right)$ and $X_{2}\left(\left|X_{2}\right|>1\right)$ are the smaller and larger $x$-distances of the two crossing points of the Nyquist locus with the $x$-axis. Since the angle is measured positive in the counter-clockwise direction, the lower phase margin is defined as $180^{\circ}-\sin ^{-1}\left(Y_{1}\right)$ and the upper phase margin is defined as $\sin ^{-1}\left(Y_{2}\right)-180^{\circ}$, where $Y_{1}$ (positive) and $Y_{2}$ (negative) are, respectively, the $y$-coordinates of the intersecting points between the Nyquist locus and the unit circle centred at origin.

For the one-sensor model, the gain and phase margins are too small. At $R a=R a^{*}=$ $10 R a_{c 0}$, for example, the margins are typically about $0.5 \mathrm{~dB}$ and $4^{\circ}$. In engineering applications, margins below $3 \mathrm{~dB}$ and $10^{\circ}$ are often considered marginal. Therefore, we conclude that as the system is stabilized for higher values of $R a$, the magnitude of the gain increases, increasing the sensitivity, as indicated by the very small gain and phase margins. Sensitivity can be reduced by implementing multiple sensors, as indicated by the improved gain and phase margins (see next subsections). 
(ii) Two-sensor control

To eliminate the lower unstable region shown in figure 6(a), we place two sensors on opposite sides of the mid-plane at $z_{s}= \pm 0.01$. It is crucial that both sensors are close to the mid-plane. Placement of one sensor or both away from the mid-plane will give rise to a lower unstable region.

In the two-sensor model we re-design the controller at the nominal condition used for the case shown in figure $6(a)$. The two-sensor model result is shown in figure $6(b)$, in which the same dashed curve as in figure $6(a)$ is included for comparison. We observe that the lower unstable region has disappeared. The neutral curve of the monotonic mode (heavy solid line) terminates at $k \approx 7.3$. Beyond this wavenumber the neutral curve of an oscillatory mode replaces the stability limit (thin solid line). If we allow $z_{s}$ to tend to zero, then the solid curve in figure $6(b)$ will approach the dashed curve. The gap between the two curves indicates that there is a trade-off between the large pole shifts due to the small sensor-plane offset, and the information gained by adding one more sensor near to the mid-plane. The gain and phase margins increase by roughly $10 \%$ to $20 \%$ by adding the second sensor. However, the improvements are still too small to be considered acceptable.

Better gain and phase margins (over $100 \%$ increase) can be obtained with sensors placed further away from the mid-plane. The sensors located at about $z_{s}= \pm 0.25$ appear to give the best result. However, in this case a lower unstable region forms. The two-sensor model is still not suited for practical implementation. For this reason, we shall not devote more effort to analysing this case. Instead, we proceed to the three-sensor model.

(iii) Three-sensor control

When three sensors are used, we can improve gain and phase margins by placing two outer sensors further away from the mid-plane without inducing any lower unstable region, provided that the remaining sensor is placed at the mid-plane. With two sensors placed significantly away from the mid-plane, it is observed that the sensor located at the mid-plane is no longer sensitive to a small offset. To determine the best sensor locations, we first observe that a lower unstable region always occurs when no sensor is placed at or very close to the mid-plane. With a mid-plane sensor in place, then by fixing one outer sensor and moving the other, it appears that the best locations are when the two outer sensors are at equal distance from the mid-plane. The best locations are determined in terms of the minimum of the real part of the least-stable closed-loop pole. Hence, for our design, we let the three sensor locations be $z_{s}^{(1)}=-z_{s}, z_{s}^{(2)}=0$ and $z_{s}^{(3)}=z_{s}$.

In order to improve gain and phase margins, we consider the Nyquist plots for various values of $z_{s}$. In the subsequent results concerning the stability limit of the controlled system (see figure 8), a good nominal condition is found to be $k^{*}=3.15$ and $R a^{*}=14.8$. For this nominal condition, figure $7(a)$ shows the Nyquist curves for $z_{s}=0.1$ (dotted), 0.2 (dashed) and 0.3 (solid). Figure $7(b)$ provides a magnified view of figure $7(a)$ near the point $(-1,0)$. The case $z_{s}=0.3$ presents no lower phase margin but has an upper phase margin of about $20.5^{\circ}$. The upper and lower gain margins are about $3.3 \mathrm{~dB}$ and $4.4 \mathrm{~dB}$, respectively. These values of gain and phase margins are quite satisfactory. A slight improvement of the margins is still possible by increasing $z_{s}$ further, at the expense of increasing the real part of the least-stable pole closer to zero, thus making the system less stable. Thus, $z_{s}=0.3$ appears to be our best choice. It is desirable to see how changing the values of $k^{*}$ and $R a^{*}$ will affect the gain and phase margins for $z_{s}=0.3$. In figure 7(c) we compare the Nyquist curves for three different nominal conditions: $k^{*}=3.15$ and $R a^{*}=14.8 R a_{c 0}$ (solid), 

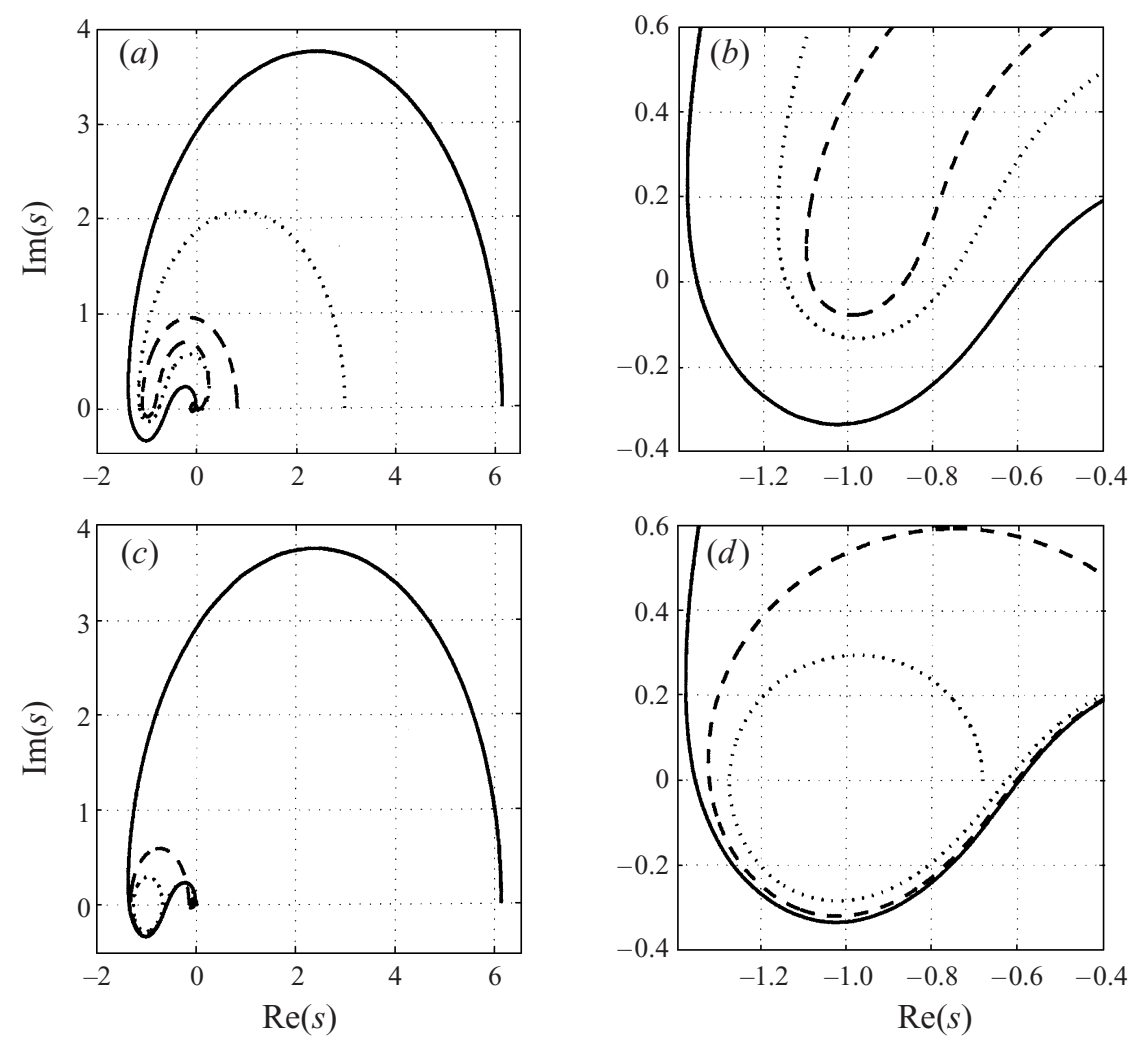

FiguRE 7. Nyquist plots for the three-sensor model: $(a)$ at nominal condition $k^{*}=3.15$ and $R a^{*}=14.5 R a_{c 0}$ and sensor locations are $z_{s}=0.1$ (dashed), 0.2 (dotted) and 0.30 (solid); $(b)$ magnified view of $(a) ;(c)$ at $z_{s}=0.3$ and nominal conditions with $k^{*}=3.15$ and $R a^{*}=14.5 R a_{c 0}$ (solid), $k^{*}=4.5$ and $R a^{*}=12.5 R a_{c 0}$ (dashed) and $k^{*}=6.5$ and $R a^{*}=15 R a_{c 0}$ (dotted); $(d)$ magnified view of $(c)$.

$k^{*}=4.5$ and $R a^{*}=12.5 R a_{c 0}$ (dashed) and $k^{*}=6.5$ and $R a^{*}=15 R a_{c 0}$ (dotted). This choice of nominal points is based on the subsequent analysis of the stability limit of the controlled system. As shown in the magnified view of figure $7(d)$, the upper phase margin and the upper and lower gain margins for the three nominal conditions are quite close, suggesting that these margins are not sensitive to the values of $k^{*}$ and $R a^{*}$. However, the lower phase margin decreases rapidly as $k^{*}$ and $R a^{*}$ increase, as shown by the dotted line. The gain and phase margins for the design case (solid) are within values used in practice.

Now, we consider the stability limit of the controlled system. In order to understand how the choice of the nominal condition $\left(k^{*}, R a^{*}\right)$ affects stability, we present the results for each set of nominal condition in figures $8(a)-8(c)$.

For each nominal point the stable region is delimited by the neutral curve. Our goal is to maximize the minimum of the neutral curve by appropriately choosing the nominal point. In figure $8(a)$ we consider $k^{*}=3$ and $R a^{*}=15 R a_{c 0}$. There is no unstable region to the left of this nominal point and the neutral curve to its right corresponds to an oscillatory mode. The neutral curve in figure $8(b)$ corresponds to $k^{*}=4.5$ and $R a^{*}=12.5 R a_{c 0}$. An unstable banana-shaped region (monotonic onset) is present on each side of the main unstable region. The minimum of the main unstable region is about $R a \approx 14.5 R a_{c 0}$. We have decreased $R a^{*}$ from $15 R a_{c 0}$ to $12.5 R a_{c 0}$ 

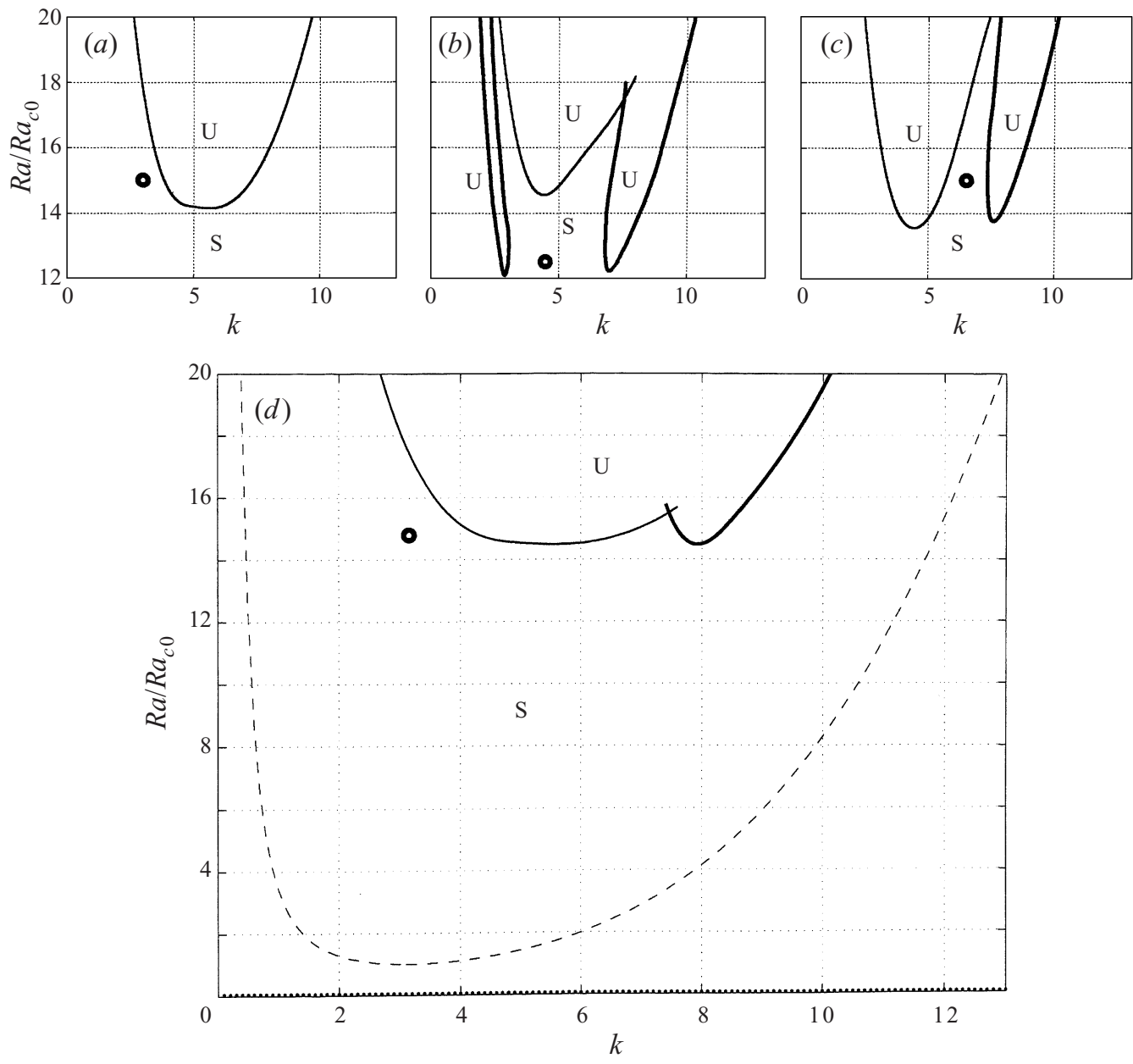

FiguRE 8 . The stability diagrams for the three-sensor model with sensor planes located at $z_{s}=-0.3$, 0 and 0.3 , and nominal conditions at $(a) k^{*}=3$ and $R a^{*}=15 R a_{c 0},(b) k^{*}=4.5$ and $R a^{*}=12.5 R a_{c 0}$, (c) $k^{*}=6.5$ and $R a^{*}=15 R a_{c 0}$. (d) $k^{*}=3.15$ and $R a^{*}=14.8 R a_{c 0}$ (the design conditions).

because at $R a^{*}=15 R a_{c 0}$ (not shown) the two banana-shaped unstable regions have merged generating a vast lower unstable region. However, because of the formation of an unstable region on each side, this nominal point is not desirable. Figure 8(c) shows the neutral curve for the system controlled by controllers designed at nominal condition $k^{*}=6.5$ and $R a^{*}=15 R a_{c 0}$. The banana-shaped region on the left of the nominal point has disappeared, but the region on the right remains.

By considering additional nominal points to the right of the first nominal point it seems that there is no significant improvement in stability. In other words, when the nominal wavenumber $k^{*}$ is larger than a certain value, the controllers become ineffective in stabilizing the entire region up to $R a=R a^{*}$, even though better local stabilization is always possible. Based on the results of figures $8(a)-8(c)$, it appears that nominal points to the right of the first point do not improve the situation. In fact, we have tried more cases involving different locations of the nominal points, but none seems to raise the minimum $R a$ of the unstable region. To achieve a maximum $R a$ for the stable range, we fine-tune the first nominal point and obtain $k^{*}=3.15$ 
and $R a^{*}=14.8 R a_{c 0}$. For this point the stability diagram is shown in figure $8(d)$. Stabilization up to $R a \approx 14.5 R a_{c 0}$ is achievable by using controllers designed at only one nominal point. The neutral curve is formed by an oscillatory mode (thin solid line) and a monotonic mode (heavy solid line). To illustrate the degree of stabilization with respect to the uncontrolled system, we include in the figure the neutral curve (dashed line) for the uncontrolled convection. Without feedback control, the region above the dashed curve is unstable to convection. Below the solid curves, however, the region is stabilized by the LQG control.

In $\S 2$, we described how in the practical implementation a controller is responsible for stabilizing an unstable normal mode whose wavenumber is indicated by a point on the $k$-axis in figure $8(d)$. Results suggest that we can use the design condition $k^{*}=3.15$ and $R a^{*}=14.8 R a_{c 0}$ for all controllers. Coincidentally, we note that the design wavenumber, $k^{*} \approx 3.15$, is quite close to the critical wavenumber of the uncontrolled convection, $k_{c 0} \approx 3.12$.

We conclude the analysis of the planar sensor model by discussing the time response of the closed-loop system. Our design condition is at $k^{*}=3.15$ and $R a^{*}=14.8 R a_{c 0}$. For high operating values of $R a$, if we turn on the controller at this design condition with no initialization of the estimator, the transient response of the controlled system induces a large actuator signal $u(t)$, which will produce actuator saturation in practical applications. Therefore, in practical applications, the operating $R a$ value should be achieved in increments of $R a$, so that for each increment the estimator remains initialized. For example, consider a controller operating at $k=k_{c} \approx 5.5$. Assume that we have increased the operating $R a$ value gradually up to $R a=12 R a_{c 0}$ so that the closed-loop system remains at the no-motion equilibrium. When approaching equilibrium, both the plant internal states and the estimator states tend to zero. As an example, we increase $R a$ from $R a=12 R a_{c 0}$ by an increment of $2 R a_{c 0}$ to the operating value $R a=14 R a_{c 0}$. Figure 9 shows the transient time responses for this case. In particular, figure $9(a)$ shows the temperatures measured by the planar sensors as functions of time, while figure $9(b)$ shows the control action signal $u(t)$. The initial transient disappears and the system settles to a new no-motion equilibrium. If we use a smaller increment than $2 R a_{c 0}$, an even better result can be expected in terms of smaller overshoot and a faster approach to equilibrium.

It is important to consider the parameters in a physical set up to see if the LQG controller can be applied to an experiment. We note that $t$ is in the unit of diffusive time, $d^{2} / \kappa$. For example, in the case of a layer of water of thickness $d=0.8 \mathrm{~cm}$, this unit is about $438 \mathrm{~s}$. A mildly supercritical condition $R a \approx 1800$ corresponds to $\Delta T^{*}=0.14^{\circ} \mathrm{C}$, while for $R a=14 R a_{c 0}$, the basic temperature difference is about $1.86^{\circ} \mathrm{C}$. Thus, the physical quantities are reasonably easy to achieve in practice.

Comparison between the stability achievable by the proportional feedback control (Tang \& Bau 1993, 1994 as well as our figure $3 a$ ) and the LQG control is possible only from a qualitative point of view. The LQG controller is more complex, due to the additional filter dynamics. The neutral curve structure is complex because the unstable regions can occur above and below the design value $R a^{*}$. However, a local stable region about $R a^{*}$ can always be maintained. In contrast, for proportional feedback there is no stable region beyond a certain value of $R a$, regardless of the gain $K_{p}$.

\subsubsection{Shadowgraph sensor model}

We now turn to the shadowgraph sensor model. The maximum $R a$ achievable over the stable range for the proportional feedback control is about $3.13 R a_{c 0}$. We attempt 

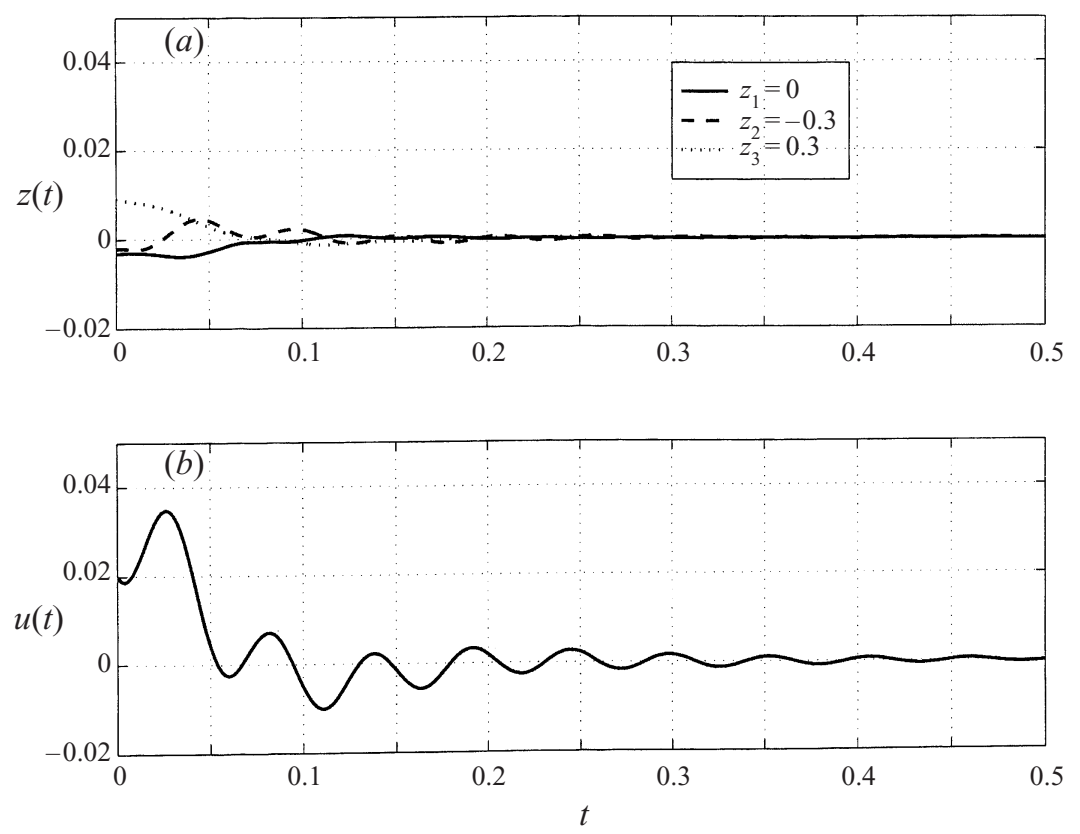

FIGURE 9. The time response of $(a)$ the three measurements and $(b)$ the control action $u(t)$, in the three-sensor model with the nominal condition $k^{*}=3.15$ and $R a^{*}=14.8 R a_{c 0}$. The convection mode considered is at $R a=14 R a_{c 0}$ and the least-stable wavenumber about $k=5.5$.

to increase the stable region using the LQG method. However, for this sensor model our results indicate a significantly weaker stabilization. We have first designed a controller for $R a^{*} \approx 10 R a_{c 0}$ but encountered a vast lower unstable region. The second convection mode, which is closest to the imaginary axis, is destabilized in the control process. As a result, we gradually decreased the nominal condition $R a^{*}$, down to a value of $5 R a_{c 0}$. The drop in performance in the critical Rayleigh number with respect to the planar sensor model is probably due to the nature of the shadowgraph sensor, which only measures the averaged temperature of the fluid layer. Figure $10(a)$ shows the stability diagram when controllers designed at five nominal points are used. The nominal points have same $R a^{*}$ while $k^{*}$ increases by a factor $\Delta k^{*}=1$. The results show that, except for the first nominal point $\left(k^{*}=1\right)$, each nominal point is enclosed by a left and a right stability limit (thin line for oscillatory mode and heavy line for monotonic mode). The stable range of wavenumbers associated with each nominal point is typically small. Figure $10(a)$ reveals two depressed unstable regions that reach below $R a=R a^{*}$ (near $k=2.4$ and $k=3.4$ ). To demonstrate how these dips can be removed, we add two more nominal points: one is chosen at $k^{*}=2.4$ and the other at $k^{*}=3.3$, both with a slightly higher $R a^{*}=5.4 R a_{c 0}$. The improved result is shown in figure $10(b)$, which indicates that the minimum $R a$ of the unstable region is now above $5 R a_{c 0}$.

Unlike the planar sensor model, the minimum $R a$ of the neutral curve obtained by applying a controller designed at a single nominal point over the whole wavenumber range is not that much higher than the value obtained using proportional feedback control. There may be further improvements on the LQG controller to be made, but we will not attempt further design in this study. 

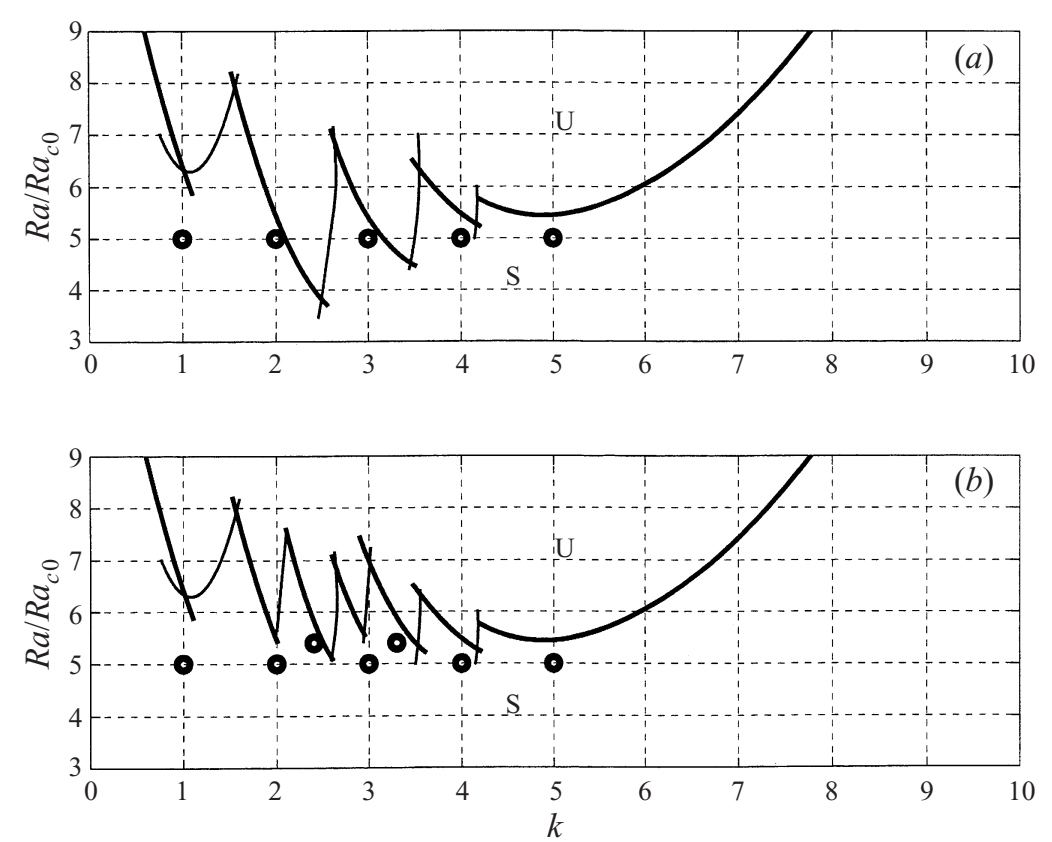

FiguRE 10. (a) The stability diagram for the shadowgraph sensor model showing the stability limit corresponding to five equally spaced nominal points at $R a^{*}=5 R a_{c 0}$. (b) An improved design with two nominal points added. Stabilization for the entire range of wavenumbers up to $R a^{*}=5 R a_{c 0}$ is achievable for this improved case.

\section{Conclusion}

We have investigated the LQG $\left(\mathscr{H}_{2}\right)$ controller design for two sensor models (planar sensor model and shadowgraph sensor model) studied by previous authors (Tang \& Bau 1994; Howle 1997a) using proportional controllers. Based on our results for $\operatorname{Pr}=7$, we have shown that the robustness of the controlled system is improved in two aspects: (i) the controller remains stable over a larger range of the parameter $R a$, and (ii) the robustness of the controller accommodates to a degree unmodelled dynamics and nonlinearities, as measured by gain and phase margins on the Nyquist diagram. It should be noted that although only one controller is needed to be designed at $\left(k^{*}, R a^{*}\right)$, this controller is implemented at each wavenumber $k$ to span the entire range of unstable wavenumbers.

The number of sensors plays an important role in dramatically improving the robustness of the stabilization of the system operating at large $R a$. Because multiple sensor planes can be easily incorporated into the planar sensor model, the performance of the planar sensor model is found to be superior to that of the shadowgraph sensor model, which only utilizes averaged temperature measurements. By using three planar sensors, it is possible to stabilize the no-motion state up to $R a \approx 14.5 R a_{c 0}$. The controller has $3 \mathrm{~dB}$ of gain margin and $20^{\circ}$ of phase margin at the design parameter values. Beyond this value of $R a$, stabilization in the region near to a nominal point can still be achieved, but an unstable region forms for $R a$ below $R a^{*}$. It should be noted that in our design procedure, we designed the controllers to span the whole range of unstable wavenumbers and at the same time demanded that the whole $R a$ range from zero up to $14.5 R a_{c 0}$ be stable. 
We have also shown that the transient responses incurred at the initial time can be reduced significantly by increasing $R a$ to its operating value in small increments. This technique allows us to initialize the estimator at each increment of $R a$ and consequently avoid controller saturations. Furthermore, by making incremental changes in $R a$ and using a controller designed to stabilize the system in a region about the design values, the value of the maximum value of $R a$ could be increased further, even though there will be unstable regions formed below stable regions in $R a$.

So far, we have assumed that the order of the controller is equal to the order of the plant. In full numerical simulations and experiments, controller designs based on reduced-order models are more practical for implementation (see, for example, Cortelezzi \& Speyer 1998; Armaou \& Christofides 2000). In our current model the actuation is assumed to be distributed continuously. In practice it will be discrete and implementation issues need to be addressed.

This research is supported by a grant from United States Air Force (Grant no. F49620-93-1-0332), and also by NASA Microgravity Physics Program (NAG3-1819) in the preliminary stage. We thank R. E. Kelly and R. T. M'Closkey for their encouragement and numerous stimulating discussions on the subject during the course of this study.

\section{REFERENCES}

Armaou, A. \& Christofides, P. D. 2000 Feedback control of the Kuramoto-Sivashinsky equation. Physica D 137, 49-61.

Bryson, A. E. \& Ho, Y. C. 1969 Applied Optimal Control. Xerox, Waltham, MA.

ChANDrasekHar, S. 1961 Hydrodynamic and Hydromagnetic Stability. Oxford University Press.

Cortelezzi, L. \& SPEYeR, J. L. 1998 Robust reduced-order controller of laminar boundary layer transitions. Phys. Rev. E 58, 1906-1910.

Cross, M. C. \& Hohenberg, P. C. 1993 Pattern formation outside of equilibrium. Rev. Mod. Phys. $\mathbf{6 5}, 851-1112$.

Doyle, J. C. \& Stein, G. 1979 Robustness with observers. IEEE Trans. Automat. Contr. AC-24, 607-611.

Gottlieb, D. \& Orszag, S. A. 1981 Numerical Analysis of Spectral Methods: Theory and Applications. SIAM, Philadelphia.

Howle, L. E. 1997a Linear stability analysis of controlled Rayleigh-Bénard convection using shadowgraphic measurement. Phys. Fluids 9, 3111-3113.

Howle, L. E. $1997 b$ Control of Rayleigh-Bénard convection in a small aspect ratio container. Intl J. Heat Mass Transfer 40, 817-822.

Howle, L. E. 1997c Active control of Rayleigh-Bénard convection. Phys. Fluids 9, 1861-1863.

Howle, L. E. 2000 The effect of boundary properties on controlled Rayleigh-Bénard convection. J. Fluid Mech. 411, 39-58.

Joshi, S. S., Speyer, J. L. \& Kim, J. 1997 A systems theory approach to the feedback stabilization of infinitesimal and finite-amplitude disturbances in plane Poiseuille flow. J. Fluid Mech. 332, 157-184.

Joshi, S. S., Speyer, J. L. \& Kim, J. 1999 Finite dimensional optimal control of Poiseuille flow. J. Guidance, Control Dyn. 22, 340-348.

KwakernaAK, H. \& Sivan, R. 1972 Linear Optimal Control Systems. John Wiley \& Sons.

Rhee, I. \& Speyer, J. L. 1991 A game theoretic approach to a finite-time disturbance attenuation problem. IEEE Trans. Automat. Contr. 36, 1021-1032.

TANG, J. \& BAU, H. H. 1993 Stabilization of the no-motion state in Rayleigh-Bénard convection through the use of feedback control. Phy. Rev. Lett. 70, 1795-1798.

TANG, J. \& BAU, H. H. 1994 Stabilization of the no-motion state in the Rayleigh-Bénard problem. Proc. R. Soc. Lond. A 447, 587-607. 
TANG, J. \& BAU, H. H. 1998 a Experiments on the stabilization of the no-motion state of a fluid layer heated from below and cooled from above. J. Fluid Mech. 363, 153-171.

TANG, J. \& BAU, H. H. $1998 b$ Numerical investigation of the stabilization of the no-motion state of a fluid layer heated from below and cooled from above, Phys. Fluids 10, 1597-1610.

Turan, L., Mingori, D. L. \& Goodwin, G. C. 1994 Loop transfer recovery design using biased and unbiased controllers. IEEE Trans. Automat. Contr. 39, 601-605. 